\title{
Phase structure and the effective potential at fixed charge
}

\author{
Katherine M. Benson* \\ Lyman Laboratory of Physics, Harvard University, Cambridge, Massachusetts 02138 \\ Jeremy Bernstein \\ Theory Division, CERN 1211 Geneva 23, Switzerland \\ Scott Dodelson ${ }^{\dagger}$ \\ Gordon McKay Laboratory, Harvard University, Cambridge, Massachusetts 02138
}

(Received 27 June 1991)

\begin{abstract}
We calculate exactly the one-loop finite-temperature effective potential for a self-interacting theory with a fixed $U(1)$ charge. We analyze in detail two limits of this exact expression: the very-low- and very-high-temperature regimes. In the former we reproduce many standard results of the nonrelativistic Bose gas. In the high-temperature limit, we describe the effects, which can be striking, of nonzero charge on vacuum structure and critical temperatures. Generally we find that in order to accommodate the fixed charge a system tends to remain longer in a spontaneously broken phase.
\end{abstract}

\section{INTRODUCTION}

Much work has been done [1] exploring how field theories behave at finite temperature. In this work, we explore the effect of a finite charge [2],[3] on a field theory. By "charge," we mean that, due to global symmetries, field theories often possess conserved quantities which remain constant at all times. We would like to understand how a nonzero charge affects the phase structure of a field theory. Do the phase transitions predicted by finite-temperature field theory still take place? Are they altered quantitatively? That is, will the presence of a nonzero charge change the critical temperature of a theory? Are there instances in which the phase structure is altered qualitatively? Does the presence of charge induce new phase transitions?

There is a textbook [4] explanation of the effect of charge on the phase structure of a system. The example used is a nonrelativistic ideal Bose gas, wherein the number of atoms in a box of volume $\Omega$ is

$$
N=\sum_{\mathbf{k}} \frac{1}{\exp \left[\frac{1}{T}\left[\left(\mathbf{k}^{2} / 2 m\right)-\mu_{\mathrm{NR}}\right]\right]-1},
$$

where the sum is over all momentum modes $\mathbf{k}=2 \pi \mathbf{n} / \Omega^{1 / 3}$ (the vector $\mathbf{n}$ is a set of three integers), $T$ is the temperature, and $\mu_{\mathrm{NR}}$ is the nonrelativistic chemical potential. In the limit that the box gets large, the sum becomes an integral as long as we first separate out the $k=0$ modes:

$$
\begin{aligned}
N= & \frac{1}{e^{-\mu_{\mathrm{NR}} / T}-1} \\
& +\Omega \int \frac{d^{3} k}{(2 \pi)^{3}} \frac{1}{\exp \left[\frac{1}{T}\left[\left(k^{2} / 2 m\right)-\mu_{\mathrm{NR}}\right]-1\right.} .
\end{aligned}
$$

This is an implicit equation for $\mu_{\mathrm{NR}}$ as a function of $T$. At high $T$ the "charge" (i.e., the number of atoms) lies in the thermally excited states. The chemical potential $\mu_{\mathrm{NR}}$ is negative and not too close to zero so the number of atoms in the ground state is of order one-insignificant compared to the number in excited states. As the temperature drops, though, $\mu_{\mathrm{NR}}$ is driven closer and closer to zero. Finally at a critical temperature $T_{c}$ determined by

$$
N=\Omega \int \frac{d^{3} k}{(2 \pi)^{3}} \frac{1}{e^{k^{2} / 2 m T_{c}}-1}
$$

the charge can no longer be accommodated by thermal modes and must reside in the ground state (in field theory the vacuum). This is the signal of a phase transition.

In the work that follows we will see the above argument in many different contexts: (i) the nonrelativistic context in which it was first worked out; (ii) the hightemperature limit of a spontaneously broken theory which would ordinarily (in the absence of charge) undergo symmetry restoration at high $T$; and (iii) the high- $T$ limit of a theory that would ordinarily have no phase structure. We will show that in all these cases, the fundamental question of "where is the charge stored" affects the quantitative and sometimes the qualitative phase structure of the theory. If the charge cannot be stored in excited modes, it must reside in the vacuum, and this is a sign that the expectation value of a charged field is nonzero, i.e., that the symmetry is spontaneously broken.

In Sec. II, we set up the formalism for evaluating the effective potential at finite temperature and charge. Section III presents a derivation of an exact expression [3] for the one-loop effective potential of the $\lambda|\phi|^{4}$ theory of a complex scalar field. In Sec. IV, we discuss the infinities in the effective potential and their renormalization. In Sec. V, we take the nonrelativistic limit of this theory and reproduce many of the well-known Bose gas results. Section VI begins a discussion of the opposite 
limit: the one in which $T$ is very high. There, we derive a simple form for the high-temperature effective potential. In Sec. VII, we use this high- $T$ limit to take a qualitative look at how the phase structure of a theory is affected by nonzero charge. Section VIII examines the phase structure more carefully; we show that in some cases a nonzero charge has a dramatic effect. We find, for the most part, that the presence of nonzero charge accelerates the onset of symmetry breaking, sometimes drastically. We also present examples where nonzero charge entirely alters the phase structure of the theory, turning systems with manifest symmetry at high temperature into ones where either the anticipated hightemperature symmetry restoration never occurs, or a new charge-induced high-temperature symmetry breakdown appears.

That charge conservation can affect the phase evolution of a theory has been considered before. Kapusta and Haber and Weldon [2] studied the effective potential in the presence of a conserved charge as we will do in Secs. II and III. Even without getting an exact expression for the one-loop effective potential, they were able to analyze various limits. Our derivation of an exact expression takes this work a step further and provides a unified framework for discussing the physics of conserved charges.

\section{THE EFFECTIVE POTENTIAL}

In this section we derive an expression for the effective potential at finite temperature and density. This is the object which, at fixed temperature and charge, must be minimized with respect to the expectation value of the scalar field and the chemical potential. We then show that in the one-loop approximation, which is as far as we will go in this paper, the effective potential is particularly simple to calculate. Much of the material in this section has appeared in the literature before [1],[2]. We present it here for completeness.

The starting point of the calculation is the grand partition function

$$
Z\left(\beta, \Omega, J_{i}, \mu\right) \equiv \operatorname{Tr}\left\{\exp \left[-\beta\left(H^{\prime}-\mu Q\right)\right]\right\},
$$

where we have explicitly written that the grand partition function $Z$ is a function of the inverse temperature $\beta$; the volume of the system $\Omega$, external sources $J_{i}$, introduced to probe the fields, and the chemical potential $\mu$. The Hamiltonian $H^{\prime}$ consists of two pieces: the ordinary Hamiltonian $H$ and the sources coupled to all external fields

$$
H^{\prime} \equiv H-\frac{J_{i}}{\beta \Omega} \int d^{3} x \phi_{i}(x) .
$$

For now, the only thing we need to know about the Hamiltonian is that there is a global symmetry resulting in a conserved Noether charge, $Q$. To account for this conserved charge, we could evaluate $\operatorname{Tr}\left[\exp \left(-\beta H^{\prime}\right)\right]$ by summing over only those states with the given value of the conserved charge. We have chosen a different approach: the sum will be over all states, but a Lagrange multiplier, the chemical potential, times the conserved charge has been added to the effective Hamiltonian. At the end of the calculation, the charge constraint is enforced by requiring

$$
\bar{Q} \equiv\langle Q\rangle=\frac{1}{\beta} \frac{\partial}{\partial \mu} \ln Z \text {. }
$$

To proceed, we define the Legendre transformation

$$
\begin{aligned}
\Gamma\left(\bar{\phi}_{i}, \mu\right) & \equiv-\ln Z\left(J_{i}, \mu\right)-J_{i} \bar{\phi}_{i} \\
& \equiv W\left(J_{i}, \mu\right)-J_{i} \bar{\phi}_{i},
\end{aligned}
$$

where $\bar{\phi}_{i}$ are some new parameters with as yet no physical significance. Note that all of these functions depend on the temperature and the volume; from now on we will not display this dependence explicitly. The advantage of this transformation is that $\Gamma$ is not a function of the (irrelevant) sources. In order for the right-hand side of (2.4) to also not be a function of the $J_{i}$, we require

$$
\left.\frac{\partial W}{\partial J_{i}}\right|_{\mu}=\bar{\phi}_{i}
$$

This equation implicitly determines $J_{i}$ in terms of $\bar{\phi}_{i}$. Equation (2.5) also tells us the significance of the parameter $\bar{\phi}_{i}$. For, returning to the definition of the partition function (2.1), one sees that

$$
\begin{aligned}
\left.\frac{\partial W}{\partial J_{i}}\right|_{\mu} & =\frac{1}{Z} \operatorname{Tr}\left(\int d^{3} x \phi_{i}(x) \exp \left[-\beta\left(H^{\prime}-\mu Q\right)\right]\right) \\
& \equiv \frac{1}{\Omega} \int d^{3} x\left\langle\phi_{i}(x)\right\rangle_{J} \\
& \equiv\left\langle\phi_{i}\right\rangle_{J}
\end{aligned}
$$

where the subscript $J$ tells us that the expectation value is taken in the presence of the sources. Therefore, Eq. (2.5) tells us that $\bar{\phi}_{i}$ is equal to the expectation value of $\phi_{i}$ in the presence of sources. Clearly, $\left\langle\phi_{i}\right\rangle_{0}$ is the quantity of physical interest since we are interested in problems where there are no external sources.

One more relevant equation follows from the definition of the Legendre transformation. If we vary both sides of Eq. (2.4) by making a small change in $\bar{\phi}_{i}$ while keeping $\mu$ fixed, we find

$$
\delta \Gamma=\left.\left.\frac{\partial W}{\partial J_{j}}\right|_{\mu} \frac{\partial J_{j}}{\partial \bar{\phi}_{i}}\right|_{\mu} \delta \bar{\phi}_{i}-\left.\frac{\partial J_{i}}{\partial \bar{\phi}_{j}}\right|_{\mu} \delta \bar{\phi}_{j} \bar{\phi}_{i}-J_{i} \delta \bar{\phi}_{i} .
$$

If there was no fixed charge, or equivalently no chemical potential, we could stop here. The point would be that at fixed $(\beta, \Omega)$ we would want $\partial \Gamma / \partial \phi_{i}$ to be zero since it is equal to $-J_{i}$ and the case of interest is $J_{i}=0$. However, with a fixed charge an arbitrary small change in $\Gamma$ is

$$
\delta \Gamma=\left.\frac{\partial \Gamma}{\partial \bar{\phi}_{i}}\right|_{\mu} \delta \bar{\phi}_{i}+\left.\frac{\partial \Gamma}{\partial \mu}\right|_{\bar{\phi}_{i}} \delta \mu .
$$

We want to keep the charge fixed, not the chemical potential, so it is not true that the stationary points of $\Gamma$ are the physically relevant states. Rather, we make one more Legendre transform; define the free energy as 


$$
F\left(\bar{\phi}_{i}, \bar{Q}\right) \equiv \frac{1}{\beta} \Gamma(\phi, \mu)+\mu \bar{Q} .
$$

The constraint equation (2.3) immediately tells us that $F$ is independent of $\mu$. Therefore, at fixed $\bar{Q}$, the system will be in a state of equilibrium when $F$ is at its minimum.

We can collect the various definitions above and write the finite-temperature and -density effective potential as

$$
\begin{aligned}
V_{\text {eff }} & \equiv \frac{F}{\Omega} \\
& =-\frac{1}{\beta \Omega}\left(\ln Z+J_{i} \bar{\phi}_{i}\right)+\frac{\mu \bar{Q}}{\Omega} .
\end{aligned}
$$

At fixed temperature, volume, and charge, $V_{\text {eff }}$ must be minimized with respect to $\mu$ and $\bar{\phi}_{i}$. This leads to three equations (if $i$ runs from 1 to 2 ) for the three unknowns $\mu$ and $\bar{\phi}_{i}$; these can be solved to give $\mu$ and $\bar{\phi}_{i}$ in terms of the physical quantities, $\beta, \Omega, \bar{Q}$.

We now outline a systematic way of calculating $Z$. First we write the trace in Eq. (2.1) as a functional integral

$$
\begin{aligned}
Z\left[J_{i}\right]=\int\left[d \phi_{1}\right]\left[d \phi_{2}\right]\left[d \pi_{1}\right]\left[d \pi_{2}\right] \\
\quad \times \exp \left(\int_{0}^{\beta} d \tau \int d^{3} x\left[i \pi_{i} \dot{\phi}_{i}-\left(\mathcal{H}^{\prime}-\mu Q\right)\right]\right) .
\end{aligned}
$$

The functional integral is over the two real fields $\left(\phi_{1}, \phi_{2}\right)$ and their conjugate momenta $\left(\pi_{1}, \pi_{2}\right)$. Since we are investigating the system at finite temperature, the integral over Euclidean time runs only from 0 to $\beta$. The implicit sum in the first term is over $i=1,2$. The Hamiltonian density for the fields is

$$
\mathscr{H}^{\prime}=\frac{1}{2}\left(\pi_{i} \pi_{i}+\nabla \phi_{i} \cdot \nabla \phi_{i}\right)+U_{0}(\phi)-\frac{J_{i}}{\beta \Omega} \phi_{i},
$$

where $\phi^{2} \equiv \phi_{1}^{2}+\phi_{2}^{2}$. For now we do not need to consider any specific choice of the potential $U_{0}$; we require only that it is a function of $\phi^{2}$. If so, then the Hamiltonian is invariant under a global $\mathrm{SO}(2)$ symmetry. This symmetry is generated by the integral over all space of the charge density:

$$
Q \equiv \phi_{1} \pi_{2}-\phi_{2} \pi_{1}
$$

The $\pi_{1}$ and $\pi_{2}$ integrals in Eq. (2.11) are easily done by completing squares; i.e., write, for example,

$$
\begin{aligned}
i \pi_{1} \dot{\phi}_{1}-\frac{1}{2} \pi_{1}^{2}-\mu \phi_{2} \pi_{1}=-\frac{1}{2}[ & \left(\pi_{1}+\mu \phi_{2}-i \dot{\phi}_{1}\right)^{2} \\
& \left.-\left(\mu \phi_{2}-i \dot{\phi}_{1}\right)^{2}\right] .
\end{aligned}
$$

Now we shift the integration variable to get

$$
\begin{aligned}
\int\left[d \pi_{1}\right] \exp \left[\int_{0}^{\beta} d \tau \int d^{3} x\left(i \pi_{1} \dot{\phi}_{1}-\frac{1}{2} \pi_{1}^{2}-\mu \phi_{2} \pi_{1}\right)\right] \\
=\exp \left[\frac{1}{2} \int_{0}^{\beta} d \tau \int d^{3} x\left(\mu \phi_{2}-i \dot{\phi}_{1}\right)^{2}\right) \\
\quad \times \int\left[d \pi_{1}^{\prime}\right] \exp \left(-\int_{0}^{\beta} d \tau \int d^{3} x \frac{1}{2} \pi_{1}^{\prime 2}\right] .
\end{aligned}
$$

The $\pi_{1}^{\prime}$ integral produces a multiplicative constant to $Z$. (For a clear discussion of this constant piece, see Bernard in Ref. [1].) Performing the same manipulations with the $\pi_{2}$ integral we have

$$
Z\left[J_{i}\right]=N \int\left[d \phi_{1}\right]\left[d \phi_{2}\right] \exp \left(-S\left[\phi_{i}, J_{i}\right]\right),
$$

where $N$ is a constant and the action in the argument of the exponential is

$$
\begin{aligned}
S=\int_{0}^{\beta} d \tau \int d^{3} x[ & \frac{1}{2}\left[\phi_{1}^{2}+\dot{\phi}_{2}^{2}+\left(\nabla \phi_{1}\right)^{2}+\left(\nabla \phi_{2}\right)^{2}\right] \\
& +U_{0}(\phi)+\frac{J_{i}}{\Omega \beta} \phi_{i} \\
& \left.+i \mu\left(\phi_{2} \dot{\phi}_{1}-\dot{\phi}_{2} \phi_{1}\right)-\frac{\mu^{2}}{2} \phi^{2}\right] .
\end{aligned}
$$

The first two lines on the right-hand side contain the terms one usually gets when not worrying about a conserved charge. The terms on the last line represent the effects of the conserved charge. Looking ahead, we notice that $\mu^{2}$ serves as a negative-mass-squared term; i.e., we might expect spontaneous symmetry breaking when $\mu$ is greater than the mass of $\phi$.

We now expand the action around a constant field configuration: $\phi_{i}=\bar{\phi}_{i}$. There are two reasons for doing so. First, we saw above that at the end of the day $\bar{\phi}_{i}$ will turn out to be the expectation value of $\phi_{i}$. The quantum fields $\phi_{i}$ will oscillate around these vacuum values with amplitudes proportional to $\hbar$, so, in the limit that $\hbar$ is small, it is reasonable to assume that these oscillations are small. Second, we will see shortly that this expansion makes it particularly easy to invert Eq. (2.5), which determines the $J_{i}$ in terms of the $\bar{\phi}_{i}$.

Carrying out the expansion is a straightforward exercise in functional derivatives, with one subtlety. Until now we have written the potential as $U_{0}(\phi)$, i.e., in terms of the bare parameters. It is convenient to break this up into renormalized parameters and counterterms:

$$
U_{0}(\phi)=U(\phi)+U_{\mathrm{ct}}(\phi) .
$$

For example, a mass term would be broken up into

$$
m_{0}^{2} \phi^{2}=m^{2} \phi^{2}+\delta m^{2} \phi^{2}
$$

and similarly for any interaction terms $\left(\lambda \phi^{4}\right.$, etc.). Note, though, that the operators in the action containing the chemical potential do not have to be divided in this fashion: the chemical potential is not renormalized, a point we will demonstrate explicitly later. In any event, once the potential is broken up as in Eq. (2.18), it is necessary to treat the terms in $U_{\mathrm{ct}}$ as higher order in the expansion parameter than those in $U$, for any differences between $U$ and $U_{0}$ are due to quantum effects and hence are proportional to $\hbar$.

So the action in Eq. (2.17) is expanded as

$$
S=S^{(0)}+S^{(1)}+S^{(2)}+S^{(\text {higher order) }} .
$$

The zero-order term is just the action evaluated at $\bar{\phi}_{i}$ :

$$
S^{(0)}=\beta \Omega\left[U(\bar{\phi})-\mu^{2} \bar{\phi}^{2} / 2\right]+J_{i} \bar{\phi}_{i},
$$

where $\bar{\phi}^{2} \equiv \bar{\phi}_{1}^{2}+\bar{\phi}_{2}^{2}$. The first-order term in (2.20) is 


$$
S^{(1)}=\int_{0}^{\beta} d \tau \int d^{3} x \phi_{i}^{\prime}(x)\left[\left.\frac{\partial U}{\partial \phi_{i}}\right|_{\phi_{i}=\bar{\phi}_{i}}-\mu \bar{\phi}_{i}+\frac{J_{i}}{\beta \Omega}\right],
$$

where $\phi_{i}^{\prime}(x)$ are the shifted fields

$$
\phi_{i}^{\prime}(x) \equiv \phi_{i}(x)-\bar{\phi}_{i}
$$

The second-order contribution to the action is

$$
S^{(2)}=\beta \Omega U_{\mathrm{ct}}(\bar{\phi})+\frac{1}{2} \int d^{4} x d^{4} y \phi_{i}^{\prime}(x) \mathcal{M}_{i j} \phi_{j}^{\prime}(y) .
$$

The first term on the right-hand side here represents the counterterms, evaluated at $\phi_{i}=\bar{\phi}_{i}$. The elements of the two-by-two matrix $\mathcal{M}$ are the functional second derivatives of the action in Eq. (2.17) with respect to $\phi_{i}^{\prime}(x)$ and $\phi_{j}^{\prime}(y)$; these must be evaluated at $\phi_{i}=\bar{\phi}_{i}$. In the next section we will write these down explicitly.

One-loop approximation The one-loop approximation corresponds to neglecting all terms in the action higher order than $S^{(2)}$, that is, all terms more than quadratic in $\phi_{i}^{\prime}$ (and any deviation from $\phi_{i}=\bar{\phi}_{i}$ in the counterterms). In this approximation,

$W \equiv-\ln Z \simeq S^{(0)}-\ln \left[\int\left[d \phi_{1}^{\prime}\right]\left[d \phi_{2}^{\prime}\right] \exp \left(-S^{(1)}-S^{(2)}\right)\right]$.

Recall that we must choose $J_{i}$ to satisfy Eq. (2.5). So we require

$$
\begin{aligned}
\bar{\phi}_{i} & =\frac{\partial S^{(0)}}{\partial J_{i}}+\frac{\partial}{\partial J_{i}} \int\left[d \phi_{1}^{\prime}\right]\left[d \phi_{2}^{\prime}\right] \exp \left(-S^{(1)}-S^{(2)}\right) \\
& =\bar{\phi}_{i}+\frac{1}{\beta \Omega} \int_{0}^{\beta} d \tau \int d^{3} x\left\langle\phi_{i}^{\prime}(x)\right\rangle
\end{aligned}
$$

The expectation value of $\phi_{i}^{\prime}$ here is simply the functional integration over the fields with an insertion of $\phi_{i}^{\prime}$; this must be zero to satisfy the equation. However, and this is one of the main advantages of initially expanding about $\bar{\phi}_{i}$ in the partition function, there is a particularly simple choice of $J_{i}$ which ensures that $\phi_{i}^{\prime}$ is zero. We can simply choose $J_{i}$ so that $S^{(1)}$, the term linear in $\phi_{i}^{\prime}$ in the action, vanishes. Then

$$
\left\langle\phi_{i}^{\prime}\right\rangle \propto \int\left[d \phi_{1}^{\prime}\right]\left[d \phi_{2}^{\prime}\right] \phi_{i}^{\prime} \exp \left(-S^{(2)}\right) .
$$

The term in the exponential and the measure are invariant under $\phi_{i}^{\prime} \rightarrow-\phi_{i}^{\prime}$, so the functional integral vanishes: $\left\langle\phi_{i}^{\prime}\right\rangle=0$.

Having now fixed $J_{i}$, we have a manageable expression for the grand partition function at one loop, which can be inserted into Eq. (2.10) to get the effective potential

$$
V_{\mathrm{eff}} \simeq U(\bar{\phi})-\mu^{2} \bar{\phi}^{2} / 2+\mu \bar{Q} / \Omega-\frac{1}{\beta \Omega} \ln Z^{(1)},
$$

where

$$
Z^{(1)} \equiv \int\left[d \phi_{1}^{\prime}\right]\left[d \phi_{2}^{\prime}\right] \exp \left(-\frac{1}{2} \int d^{4} x d^{4} y \phi_{i}^{\prime}(x) M_{i j} \phi_{j}^{\prime}(y)\right) \text {. }
$$

Note that this expression for $Z^{(1)}$ is exactly what we would have gotten had we started naively without introducing any sources. We would have expanded about the constant fields $\bar{\phi}_{i}$ and kept only quadratic terms. The formalism introduced in this section has three purposes: (i) it convinces us that we must minimize the full one-loop expression for $V_{\text {eff }}$, not just the tree-level piece, with respect to $\bar{\phi}_{i}$; (ii) it convinces us that $\left\langle\phi_{i}\right\rangle=\bar{\phi}_{i}$; and (iii) it provides a prescription, or at least a starting point, for going further and calculating higher-order corrections.

\section{THE ONE-LOOP EFFECTIVE POTENTIAL}

With the formalism of the previous section, we can now compute the one-loop effective potential. We have expanded the action in Eq. (2.17) about the constant field configuration $\bar{\phi}_{i}$ and kept only terms up to quadratic in $\phi_{i}-\bar{\phi}_{i}$. The linear terms are chosen to vanish with an appropriate choice of the sources $J_{i}$.

It remains to calculate the coefficients of the quadratic terms, i.e., the elements of the $2 \times 2$ matrix $M_{i j}$ in Eq. (2.29). These are the second functional derivatives of the action with respect to the fields evaluated at $\bar{\phi}_{i}$,

$$
\left.\mathcal{M}_{i j} \equiv \frac{\delta^{2} S}{\delta \phi(x) \delta \phi(y)}\right|_{\phi_{i}=\bar{\phi}_{i}}
$$

so a simple exercise in functional differentiation yields

$$
\begin{aligned}
& M_{11}=\delta^{4}(x-y)\left[-\partial_{\mu} \partial_{\mu}-2 \mu^{2}+U^{\prime \prime} \frac{\phi_{1}^{2}}{\phi^{2}}+\frac{U^{\prime}}{\phi} \frac{\phi_{2}^{2}}{\phi^{2}}\right]_{\phi_{i}=\bar{\phi}_{i}}, \\
& M_{12}=\delta^{4}(x-y)\left[2 i \mu \frac{\partial}{\partial \tau}+U^{\prime \prime} \frac{\phi_{1} \phi_{2}}{\phi^{2}}-\frac{U^{\prime}}{\phi} \frac{\phi_{1} \phi_{2}}{\phi^{2}}\right]_{\phi_{i}=\bar{\phi}_{i}}, \\
& M_{21}=\delta^{4}(x-y)\left[-2 i \mu \frac{\partial}{\partial \tau}+U^{\prime \prime} \frac{\phi_{1} \phi_{2}}{\phi^{2}}-\frac{U^{\prime}}{\phi} \frac{\phi_{1} \phi_{2}}{\phi^{2}}\right]_{\phi_{i}=\bar{\phi}_{i}}, \\
& M_{22}=\delta^{4}(x-y)\left(-\partial_{\mu} \partial_{\mu}-2 \mu^{2}+U^{\prime \prime} \frac{\phi_{2}^{2}}{\phi^{2}}+\frac{U^{\prime}}{\phi} \frac{\phi_{1}^{2}}{\phi^{2}}\right]_{\phi_{i}=\bar{\phi}_{i}} .
\end{aligned}
$$

The apparent dependence of $\mathcal{M}$ on $\phi_{1}$ and $\phi_{2}$ separately is illusory: the final expression for $Z^{(1)}$ depends only on the combination of $\phi^{2}=\phi_{1}^{2}+\phi_{1}^{2}$. To proceed, we will now make a Fourier expansion of $\phi_{i}^{\prime}$ taking advantage of the fact that Bose statistics demand that $\phi_{i}^{\prime}(\mathbf{x}, \tau)$ is periodic in $\tau$ with period $\beta$. Thus,

$$
\phi_{i}^{\prime}(\mathbf{x}, \tau)=\beta^{1 / 2} \sum_{n=-\infty}^{\infty} \phi_{i, n}^{\prime}(\mathbf{x}) e^{i \omega_{n} \tau}
$$

with

$$
\omega_{n}=\frac{2 \pi n}{\tau} \text {. }
$$

In $\mathcal{M}$, each $\tau$ derivative is now replaced by $i \omega_{n}$ and the integration over $\tau$ in the exponential of $Z^{(1)}$ can be done trivially to get

$$
\begin{aligned}
Z^{(1)}=\int & {\left[d \phi_{1, n}^{\prime}\right]\left[d \phi_{2, n}^{\prime}\right] } \\
& \times \exp \left[-\frac{1}{2} \sum_{n} \int d^{3} x \phi_{i, n}^{\prime}(\mathbf{x}) \mathcal{M}_{i j}^{\prime} \phi_{j, n}^{\prime}(\mathbf{x})\right],
\end{aligned}
$$

where $\mathcal{M}_{i j}^{\prime}$ is just $M_{i j} \beta^{2}$ with the $\delta$ function removed and $\partial / \partial \tau \rightarrow i \omega_{n}$. We may now use the relation 


$$
\begin{aligned}
\int\left[d \phi_{1, n}^{\prime}\right]\left[d \phi_{2, n}^{\prime}\right] & \exp \left(-\frac{1}{2} \sum_{n} \int d^{3} x \phi_{i, n}^{\prime}(\mathbf{x}) \mathcal{M}_{i j}^{\prime} \phi_{j, n}^{\prime}(\mathbf{x})\right) \\
& =\frac{N^{\prime}}{\left|\operatorname{det} \mathcal{M}^{\prime}\right|^{1 / 2}} \\
& =\frac{N^{\prime}}{\left|\operatorname{det}\left(\mathcal{M}_{11}^{\prime} \mathcal{M}_{22}^{\prime}-\mathcal{M}_{12}^{\prime} \mathcal{M}_{21}^{\prime}\right)\right|^{1 / 2}}
\end{aligned}
$$

Here $N^{\prime}$ is an infinite $\beta$-independent constant that we will absorb in overall normalization of the functional integral [see Eq. (2.16)]. There still remains a determinant since each $\mathcal{M}_{i j}^{\prime}$ is an operator. We may now use the identity $\operatorname{det} A=\exp (\operatorname{Tr} \ln A)$ to bring the determinant into the exponential. We can go into momentum space to write

$$
\ln Z^{(1)}=-\frac{\Omega}{2} \sum_{n=-\infty}^{\infty} \int \frac{d^{3} k}{(2 \pi)^{3}} \ln \left\{\beta^{4}\left[\left(\omega_{n}^{2}+k^{2}\right)^{2}+4 \mu^{2} \omega_{n}^{2}+\left(\omega_{n}^{2}+k^{2}\right)\left(U^{\prime \prime}+\frac{U^{\prime}}{\phi}-2 \mu^{2}\right)+\left(U^{\prime \prime}-\mu^{2}\right)\left[\frac{U^{\prime}}{\phi}-\mu^{2}\right)\right]\right\},
$$

where $\phi$ is to be evaluated at $\bar{\phi}$ everywhere. We can rewrite this fourth-order polynomial in $\omega_{n}$ as a product of two second-order polynomials. The argument of the log is then

$$
\beta^{4}\left(\omega_{n}^{2}+i \alpha \omega_{n}+\gamma\right)\left(\omega_{n}^{2}-i \alpha \omega_{n}+\gamma\right),
$$

where

$$
\gamma^{2} \equiv\left(k^{2}+U^{\prime \prime}-\mu^{2}\right)\left(k^{2}+\frac{U^{\prime}}{\phi}-\mu^{2}\right), \quad \alpha^{2} \equiv 2\left[k^{2}+\mu^{2}+\frac{1}{2}\left(U^{\prime \prime}+\frac{U^{\prime}}{\phi}\right)-\gamma\right] .
$$

Thus we can write

$$
\begin{aligned}
\sum_{n=-\infty}^{\infty} \ln \beta^{4}\left[\left(\omega_{n}^{2}+k^{2}\right)^{2}+4 \mu^{2} \omega_{n}^{2}+\left(\omega_{n}^{2}+k^{2}\right)\right. & {\left.\left[U^{\prime \prime}+\frac{U^{\prime}}{\phi}-2 \mu^{2}\right]+\left(U^{\prime \prime}-\mu^{2}\right)\left(\frac{U^{\prime}}{\phi}-\mu^{2}\right)\right] } \\
& =\sum_{n=-\infty}^{\infty}\left\{\ln \left[\beta^{2}\left(\omega_{n}^{2}+i \alpha \omega_{n}+\gamma\right)\right]+(\alpha \rightarrow-\alpha)\right\} \\
& =\left[\ln \prod_{n=-\infty}^{\infty} \beta^{2}\left(\omega_{n}^{2}+i \alpha \omega_{n}+\gamma\right)\right]+(\alpha \rightarrow-\alpha) \\
& =\ln \prod_{n=-\infty}^{\infty} \beta^{2}\left[\omega_{n}+\frac{i \alpha}{2}\right]^{2}+\ln \prod_{n=-\infty}^{\infty}\left[1+\frac{\gamma+\alpha^{2} / 4}{\left(\omega_{n}+i \alpha / 2\right)^{2}}\right]+(\alpha \rightarrow-\alpha) .
\end{aligned}
$$

The second infinite product can be evaluated with the aid of the identity

$$
\prod_{n=-\infty}^{\infty}\left(1+\frac{\beta^{2}\left(\gamma+\alpha^{2} / 4\right)}{(2 \pi n+i \alpha \beta / 2)^{2}}\right)=\frac{\cosh \left(\beta \sqrt{\gamma+\alpha^{2} / 4}\right)-\cosh (\alpha \beta / 2)}{1-\cosh (\alpha \beta / 2)} .
$$

The cosh's in this term ensure that it is independent of the sign of $\alpha$ so the $-\alpha$ term in Eq. (3.10) gives an equal contribution. The first term on the right-hand side (3.10) can be combined with its $-\alpha$ counterpart to give

$2 \ln \prod_{n=-\infty}^{\infty} \beta^{2}\left[\omega_{n}^{2}-(\alpha / 2)^{2}\right]=2 \ln \left(\frac{\beta^{2} \alpha^{2}}{4}\right)+4 \ln \prod_{n \geq 1}\left(\beta \omega_{n}\right)^{2}+4 \ln \prod_{n \geq 1}\left[1+\left(\frac{\beta \alpha}{4 \pi n}\right)^{2}\right]=4 \ln \sinh (\alpha \beta / 4)+4 \ln \prod_{n \geq 1}\left(\beta \omega_{n}\right)^{2}$.

The last term here is an infinite constant which we incorporate into our overall constant collector $N$. The first term exactly cancels $(1-\cosh )$ denominator in Eq. (3.11) so that the whole sum over $n$ in Eq. (3.10) reduces to the difference of two cosh's. Finally using $\cosh x-\cosh y=\frac{1}{2} e^{-x}[1-\exp (x+y)][1-\exp (x-y)]$, we arrive at the principal result of this section: i.e.,

$$
V_{\text {eff }}=\left(\frac{\text { const }}{\beta \Omega}\right)+V_{\text {tree }}+V_{\text {thermal }}+V_{\text {zero point }},
$$

with the tree-level contribution

$$
V_{\text {tree }} \equiv U(\bar{\phi})-\frac{1}{2} \mu^{2} \bar{\phi}^{2}+\mu \frac{\bar{Q}}{\Omega}+U_{\mathrm{ct}}(\bar{\phi}),
$$

thermal contribution 


$$
V_{\text {thermal }} \equiv \frac{1}{\beta} \int \frac{d^{3} k}{(2 \pi)^{3}} \ln \left(1-e^{-\beta E_{+}(k)}\right)\left(1-e^{-\beta E_{-}(k)}\right),
$$

and zero-point energy

$$
V_{\text {zero point }} \equiv \int \frac{d^{3} k}{(2 \pi)^{3}} \frac{E_{+}(k)+E_{-}(k)}{2}
$$

The excitation energies in Eqs. (3.15) and (3.16) are

$$
\begin{aligned}
E_{ \pm}(k)^{2} & \equiv \sqrt{\gamma+\alpha^{2} / 4} \pm \frac{\alpha}{2} \\
& =k^{2}+\mu^{2}+\frac{1}{2}\left[U^{\prime \prime}(\bar{\phi})+\left.\frac{U^{\prime}}{\phi}\right|_{\phi=\bar{\phi}}\right] \pm\left[2 \mu^{2}\left[2 k^{2}+U^{\prime \prime}(\bar{\phi})+\left.\frac{U^{\prime}}{\phi}\right|_{\phi=\bar{\phi}}\right]+\frac{1}{4}\left[U^{\prime \prime}(\bar{\phi})-\left.\frac{U^{\prime}}{\phi}\right|_{\phi=\bar{\phi}}\right]^{2}\right]^{1 / 2} .
\end{aligned}
$$

In Ref. [3], we explored the form of these excitation energies in various limits. For example, if $\bar{\phi}=0$ then the excitation energies are simply $E_{ \pm}=\sqrt{k^{2}+m^{2}} \pm \mu$. In the presence of a vacuum expectation value for $\phi$, though, the excitation energies change as demonstrated in [3]. The zero-point energy, Eq. (3.16), is infinite; the infinities must be canceled by terms in $U_{\mathrm{ct}}$. In the next section we treat these infinities; specifically we show that the infinite parts of the zero-point energy are independent of the chemical potential $\mu$. Finally we mention here that the Nernst theorem requires the constant in Eq. (3.13) to be 0 , as we will argue in the nonrelativistic section.

The expression (3.13) for the effective potential is valid for a general potential $U(\phi)$. In the remainder of this paper we will consider the simplest example for $U$, the $\phi^{4}$ potential:

$$
U(\phi)=\frac{1}{2} m^{2} \phi^{2}+\frac{\lambda}{4 !} \phi^{4} .
$$

More complicated forms for $U$ have been considered recently [5] but there is much interesting physics lurking in the $\phi^{4}$ theory and we will restrict our attention to this case. The excitation energies now become

$E_{ \pm}(k)^{2}=k^{2}+\widetilde{m}^{2}+\mu^{2} \pm \sqrt{4 \mu^{2}\left(k^{2}+\tilde{m}^{2}\right)+m^{4} \delta^{2}}$

where we have defined

$$
\delta \equiv \frac{\lambda}{6 m^{2}} \bar{\phi}^{2}
$$

and

$$
\widetilde{m}^{2} \equiv m^{2}(1+2 \delta) \text {. }
$$

For future use, we note the following identities involving $E_{ \pm}:$

$$
E_{+} E_{-}=\sqrt{\left(k^{2}+\tilde{m}^{2}-\mu^{2}\right)^{2}-m^{4} \delta^{2}}
$$

and therefore

$$
\begin{aligned}
E_{+}+E_{-}=\sqrt{2} & {\left[k^{2}+\tilde{m}^{2}+\mu^{2}\right.} \\
& \left.+\sqrt{\left(k^{2}+\tilde{m}^{2}-\mu^{2}\right)^{2}-m^{4} \delta^{2}}\right]^{1 / 2}
\end{aligned}
$$

We are now in a position to study the infinities.

\section{THE INFINITIES}

At the one-loop order we encounter ultraviolet divergences which arise when we perform the integration

$$
V_{\text {zero point }}=\int \frac{d^{3} k}{(2 \pi)^{3}} \frac{E_{+}(k)+E_{-}(k)}{2} .
$$

We regularize these infinities by introducing an ultraviolet cutoff $\Lambda$ and requiring $|\mathbf{k}|<\Lambda$. Then we may examine the divergent terms by writing

$$
\begin{aligned}
E_{+}(k)+E_{-}(k) & =\sqrt{2}\left[k^{2}+\widetilde{m}^{2}+\mu^{2}+\sqrt{\left(k^{2}+\tilde{m}^{2}-\mu^{2}\right)^{2}-m^{4} \delta^{2}}\right]^{1 / 2} \\
& =\sqrt{2} k\left\{1+\frac{\tilde{m}^{2}+\mu^{2}}{k^{2}}+\left[\left[1+\frac{\widetilde{m}^{2}-\mu^{2}}{k^{2}}\right]^{2}-\frac{m^{4} \delta^{2}}{k^{4}}\right]^{1 / 2}\right\}^{1 / 2}
\end{aligned}
$$

For large $k$ the square root has an expansion of the form

$$
\left\{1+\frac{\widetilde{m}^{2}+\mu^{2}}{k^{2}}+\left[\left[1+\frac{\widetilde{m}^{2}-\mu^{2}}{k^{2}}\right]^{2}-\frac{m^{4} \delta^{2}}{k^{4}}\right]^{1 / 2}\right\}^{1 / 2}=a_{0}+\frac{a_{2}}{k^{2}}+\frac{a_{4}}{k^{4}}+F\left(\mu^{2}, \widetilde{m}^{2}, \delta^{2}, k^{2}\right) \equiv I\left(\mu^{2}, \widetilde{m}^{2}, \delta^{2}, k^{2}\right),
$$

where $F\left(\mu^{2}, \widetilde{m}^{2}, \delta^{2}, k^{2}\right)$ involves terms that do not contribute to the ultraviolet divergences. As we see from Eq. (4.2) these divergences are quartic, quadratic, and logarithmic. We see by inspection that $a_{0}=\sqrt{2}$, so the quartically divergent term depends only on the cutoff $\Lambda$, i.e., it is independent of the chemical potential $\mu$. We now want to argue that $a_{2}$ and $a_{4}$ are independent of $\mu$ as well. Note first that

$$
I\left(\mu^{2}, 0,0, k^{2}\right)=\sqrt{2} .
$$


This means that there are no terms in the expansion of the form $\mu^{2} / k^{2}$ or $\mu^{4} / k^{4}$. Next consider

$$
I\left(\mu^{2}, \widetilde{m}^{2}, 0, k^{2}\right)=\sqrt{2} \sqrt{1+\left(\tilde{m}^{2} / k^{2}\right)} .
$$

This means there is no term in the expansion of the form $\mu^{2} \widetilde{m}^{2} / k^{4}$. Terms of the form $\mu^{2} m^{4} \delta^{2} / k^{6}$ do not diverge. Hence there are no divergent terms involving $\mu$.

This result could have been anticipated. Recall that the chemical potential enters the Lagrangian in the form

$$
i \mu\left(\phi_{2} \dot{\phi}_{1}-\dot{\phi}_{2} \phi_{1}\right) \equiv u_{v} J_{v}
$$

where $u^{v}$ (in Euclidean space, $v$ runs from 1 to 4 ) is an external vector potential of the form

$$
u^{v}=(0,0,0, \mu)
$$

and $J_{v}$ is the conserved current whose charge generates the transformations of the global $\mathrm{SO}(2)[=\mathrm{U}(1)]$ group. Because of this property of $J_{v}$, and its associated charge, there is a Ward identity of the form [6]

$$
-Z_{J}\left(p^{\prime}-p\right)_{v} V_{v}^{R}\left(p^{\prime}, p\right)=\Delta^{R}\left(p^{\prime}\right)-\Delta^{R}(p),
$$

where $V_{v}^{R}$ is the renormalized $J_{v}$ vertex, while $\Delta^{R}$ is the renormalized $\phi$ propagator. It follows from Eq. (4.8) that $Z_{J}$ is finite so that none of the infinities in any order can depend on $\mu$. This has the important consequence that the expectation value of the charge operator $\bar{Q} \equiv\langle Q\rangle$ is finite to all orders.

We now want to study the infinities. Since these are independent of $\mu$, we can simply set $\mu=0$ in $E_{ \pm}$and consider

$$
\begin{aligned}
V_{\text {zero point }}^{\infty} \equiv P_{I}\left(V_{\text {zero point }}\right) & \\
=P_{I}\left[\frac { 1 } { 2 } \int _ { | \mathbf { k } | < \Lambda } \frac { d ^ { 3 } k } { ( 2 \pi ) ^ { 3 } } \left(\sqrt{k^{2}+\widetilde{m}^{2}+m^{2} \delta}\right.\right. & \\
& \left.\left.+\sqrt{k^{2}+\tilde{m}^{2}-m^{2} \delta}\right)\right],
\end{aligned}
$$

where $P_{I}$ is the "infinite part," which is the part which diverges as the cutoff $\Lambda$ goes to infinity. These integrals can be evaluated analytically using the identity

$$
\begin{aligned}
\int d k k^{2} \sqrt{k^{2}+M^{2}}= & \frac{1}{4} k \sqrt{k^{2}+M^{2}}\left(k^{2}+\frac{M^{2}}{2}\right) \\
& -\frac{1}{8} M^{4} \ln \left(k+\sqrt{k^{2}+M^{2}}\right) .
\end{aligned}
$$

It is straightforward to take the limit of the resulting analytic expression as $\Lambda \rightarrow \infty$. Dropping all finite parts after taking the limit leads to

$$
\begin{aligned}
V_{\text {zero point }}^{\infty}= & {\left[\frac{\Lambda^{4}}{8 \pi^{2}}+\frac{\Lambda^{2} m^{2}}{8 \pi^{2}}+\frac{m^{4}}{16 \pi^{2}} \ln (\kappa / \Lambda)\right] } \\
& +\left[\frac{\lambda \Lambda^{2}+\ln (\kappa / \Lambda)}{24 \pi^{2}} \bar{\phi}^{2}\right]+\left(\frac{5 \lambda^{2} \ln (\kappa / \Lambda)}{(4 ! \pi)^{2}} \bar{\phi}^{4}\right),
\end{aligned}
$$

where $\kappa$ is an arbitrary scale introduced to account for our freedom to add and subtract finite pieces from

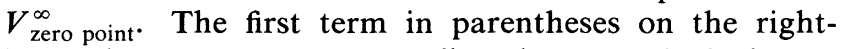
hand side serves to renormalize the cosmological constant, the second to renormalize the mass, and the third, the coupling constant.

As an example of the renormalization process, and as a check on our calculation, we can use the last term in Eq. (4.11) to find the $\beta$ function for the theory. Recall that this is defined as

$$
\beta \equiv \kappa \frac{d \lambda}{d \kappa}
$$

To find how $\lambda$ depends on $\kappa$, we note that this infinity must be canceled by the counterterms. Specifically we require

$$
\frac{\lambda_{0}-\lambda}{4 !} \bar{\phi}^{4}+\frac{5 \lambda^{2} \ln (\kappa / \Lambda)}{(4 ! \pi)^{2}} \bar{\phi}^{4}=\text { finite . }
$$

But the bare coupling $\lambda_{0}$ does not depend on the arbitrary renormalization scale $\kappa$, so we can find $d \lambda / d \kappa$ by differentiating Eq. (4.13) with respect to $\kappa$. To the requisite order

$$
-\frac{1}{4 !} \frac{d \lambda}{d \kappa}+\frac{5 \lambda^{2}}{(4 ! \pi)^{2} \kappa}=0
$$

leading immediately to the correct expression for the $\beta$ function:

$$
\beta=\frac{5 \lambda^{2}}{24 \pi^{2}} .
$$

\section{NONRELATIVISTIC LIMIT}

The one-loop expression for the effective potential, Eq. (3.13), is compact but it is difficult to use in actual computations. In this section we explore the nonrelativistic limit of the effective potential. We will see that, in this limit, Eq. (3.13) becomes much more manageable, and many of the standard results of the nonrelativistic Bose gas [4] can be retrieved.

What is the nonrelativistic limit? As an example, consider liquid ${ }^{4} \mathrm{He}$. The mass of a helium atom is roughly $4 m_{p}=4 \mathrm{GeV}$, while typical temperatures are of order several degrees Kelvin, i.e., $T \sim 4 \times 10^{-4} \mathrm{eV}$. So $T / m \sim 10^{-13}$. The temperature governs the magnitude of the kinetic energy, so we expect $k^{2} / 2 m$ to be of order $T$, leading to the first definition

$$
x \equiv \frac{k^{2}}{2 m^{2}} .
$$

This quantity should surely be very small. The amount of energy needed to add a particle to the system is equal to the chemical potential. In the nonrelativistic limit, therefore we expect $\mu=m+O(T)$, leading to the next definition.

$$
y \equiv \frac{\mu_{\mathrm{NR}}}{m} \equiv \frac{\mu-m}{m} .
$$

Thus, $y$ should be of order $x$, i.e., of order $10^{-13}$. Finally, 
we will see that another dimensionless quantity of the same order of magnitude as $x, y$, is $\delta$ defined in Eq. (3.20) as $\lambda \bar{\phi}^{2} / 6 m^{2}$.

We can immediately write the tree-level part of $V_{\text {eff }}$ in terms of the small parameters defined above, so that

$$
V_{\mathrm{tree}}^{\mathrm{NR}}=\frac{6 \delta m^{4}}{\lambda}\left(-y+\frac{\delta}{4}\right)+m n(1+y)+U_{\mathrm{ct}},
$$

where $n \equiv \bar{Q} / \Omega$ is the number density and we have set $-y-y^{2} / 2 \rightarrow-y$ in accord with the nonrelativistic limit. We will shortly derive expressions for $V_{\text {thermal }}$ and $V_{\text {zero point }}$ in this limit, but first we want to solve the zero-order equations for $\bar{\phi}$ and $\mu$, or equivalently for $\delta$ and $y$. The effective potential must be minimized with respect to $\bar{\phi}$ and $\mu$, so in terms of $\delta$ and $y$ we require

$$
\begin{aligned}
& 0=\delta \frac{\partial V_{\mathrm{eff}}^{\mathrm{NR}}}{\partial \delta} \Longrightarrow y_{0}=\delta_{0} / 2, \\
& 0=\frac{\partial V_{\mathrm{eff}}^{\mathrm{NR}}}{\partial y} \Longrightarrow \delta_{0}=\frac{\lambda n}{6 m^{3}} .
\end{aligned}
$$

The subscripts 0 here refer to the fact that these equations were derived from the tree-level terms in $V_{\mathrm{eff}}^{\mathrm{NR}}$. However, even at this simple level, two points are worth noting. First, we see that if the number density is nonzero, then $\delta_{0}=0$ is not a solution to the equations; that is, $\bar{\phi}$ is always nonzero in this approximation. This is reasonable since we have neglected thermal effects: all the charge must be stored in the ground state, i.e., in the $k=0$ mode. This vacuum charge is $\mu \bar{\phi}^{2} \rightarrow m \bar{\phi}^{2}$ in the nonrelativistic limit, so $\bar{\phi}$ must be nonzero. In other words, in the absence of thermal excitations, a bosonic system must condense in order to store the charge. The second point about the zero-order equations (5.4) is that the dimensionless parameter $\delta$ is indeed typically very small. To see this, it is convenient to introduce the scattering length

$$
a \equiv \frac{\lambda}{48 \pi m} \text {. }
$$

In terms of $a, \delta_{0}$ is equal to $8 \pi n a / m^{2}$. For liquid ${ }^{4} \mathrm{He}$ $n^{-1} \simeq 45 \AA^{3}, a \simeq 2.2 \AA$, and $m \simeq 4 \mathrm{GeV}=2 \times 10^{6} \AA^{-1}$, so $\delta_{0} \simeq 3 \times 10^{-13}$.

Now let us consider the thermal piece $V_{\text {thermal }}$ in the effective potential. In terms of the dimensionless variables above, the expression for this contribution, Eq. (3.15), becomes

$$
V_{\text {thermal }}^{\mathrm{NR}}=\frac{m^{3}}{\sqrt{2} \pi^{2} \beta} \int_{0}^{\infty} d x x^{1 / 2} \ln \left(1-e^{-\beta E_{-}^{\mathrm{NR}}}\right) .
$$

In the nonrelativistic limit, $E_{+} \simeq 2 m$, so the contribution from $\ln \left(1-e^{-\beta E_{+}}\right)$is suppressed by a factor of $e^{-2 m / T}$. Thus we have kept only the $E_{\text {_ }}$ piece. The nonrelativistic limit of $E_{-}$can be derived by a simple Taylor expansion of Eq. (3.17) in the small quantities $x, y, \delta$ :

$$
E_{-}^{\mathrm{NR}}=m\left[(x-y+\delta)^{2}-\frac{\delta^{2}}{4}\right]^{1 / 2} \text {. }
$$

The zero-point contribution to the effective potential, Eq. (3.16), can also be written in the nonrelativistic limit:

$$
V_{\text {zero point }}^{\mathrm{NR}}=\frac{m^{4}}{\sqrt{2} \pi^{2}} \int_{0}^{\Lambda^{2} / 2 m^{2}} d x x^{1 / 2}\left[1+\frac{y+x+\delta}{2}+\frac{1}{2}\left[(x+\delta-y)^{2}-\delta^{2} / 4\right]^{1 / 2}\right]
$$

This expression diverges as $\Lambda \rightarrow \infty$; the divergences must be canceled by terms in $U_{\mathrm{ct}}$. Specifically, we can add and subtract $x^{1 / 2}\left(1+x+\delta-\delta^{2} / 16 x\right)$ to the integrand in Eq. (5.8). This leads to

$$
\begin{aligned}
V_{\text {zero point }}^{\mathrm{NR}}= & \frac{m^{4}}{2 \sqrt{2} \pi^{2}} \int_{0}^{\Lambda^{2} / 2 m^{2}} d x x^{1 / 2}\left[\left[(x+\delta-y)^{2}-\delta^{2} / 4\right]^{1 / 2}-(x+\delta-y)+\frac{\delta^{2}}{8 x}\right) \\
& +\frac{m^{4}}{\sqrt{2} \pi^{2}} \int_{0}^{\Lambda^{2} / 2 m^{2}} d x x^{1 / 2}\left(1+x+\delta-\frac{\delta^{2}}{16 x}\right) .
\end{aligned}
$$

The term on the first line is finite as $\Lambda \rightarrow \infty$, while the infinities in the second term must be absorbed by the counterterms in $U_{\mathrm{ct}}$. Parenthetically, we note that this renormalization scheme leads us to set

$$
0=\frac{\lambda_{0}-\lambda}{4 !} \bar{\phi}^{4}-\frac{m^{2} \delta^{2}}{16 \sqrt{2} \pi^{2}} \int_{0}^{\Lambda^{2} / 2 m^{2}} \frac{d x}{x^{1 / 2}}
$$

or

$$
\lambda=\lambda_{0}\left(1-\frac{\lambda_{0}}{24 \pi^{2}} \frac{\Lambda}{m}\right) .
$$

The fact that the correction to $\lambda$ grows linearly with the cutoff $\Lambda$ in the nonrelativistic limit has been noted and discussed by Beg and Furlong [7] and recently by Jackiw [8].

We can now write the full one-loop expression for the effective potential in the nonrelativistic limit 


$$
\begin{aligned}
\frac{1}{m^{4}} V_{\text {thermal }}^{\mathrm{NR}}= & \frac{6 \delta}{48 \pi m a}\left[\frac{\delta}{4}-y\right]+n(1+y) / m^{3}+\frac{1}{2 \sqrt{2} \pi^{2}} \int_{0}^{\Lambda^{2} / 2 m^{2}} d x x^{1 / 2}\left[\left[(x+\delta-y)^{2}-\delta^{2} / 4\right]^{1 / 2}-(x+\delta-y)+\frac{\delta^{2}}{8 x}\right] \\
& +\frac{1}{\sqrt{2} \pi^{2} \beta m} \int_{0}^{\infty} d x x^{1 / 2} \ln \left(1-\exp \left\{-\beta m\left[(x+\delta-y)^{2}-\delta^{2} / 4\right]\right\}\right)
\end{aligned}
$$

There are two well-known results which emerge immediately from the nonrelativistic limit of the effective potential, Eq. (5.12). First, we can find the number density of particles in excited states even at zero temperature due to quantum effects. This is just

$$
\begin{aligned}
n_{\mathrm{zero} \text { point }}^{\mathrm{NR}} & =-\frac{\partial}{\partial \mu} V_{\text {zero point }}^{\mathrm{NR}}=-\frac{1}{m} \frac{\partial}{\partial y} V_{\text {zero point }}^{\mathrm{NR}} \\
& =\frac{m^{3}}{2 \sqrt{2} \pi^{2}} \int_{0}^{\infty} d x x^{1 / 2}\left[\frac{x+\delta-y}{\left[(x+\delta-y)^{2}-\delta^{2} / 4\right]^{1 / 2}}-1\right] .
\end{aligned}
$$

To recapture the standard result, we use the tree-level expression for $\delta$ and $y$, Eq. (5.4), to get

$$
\begin{aligned}
n_{\mathrm{zero} \text { point }}^{\mathrm{NR}} & =\frac{m^{3}}{2 \sqrt{2} \pi^{2}} \int_{0}^{\infty} d x x^{1 / 2}\left(\frac{x+y_{0}}{\left(x^{2}+2 x y_{0}\right)^{1 / 2}}-1\right) \\
& =\frac{8}{3 \sqrt{\pi}}(n a)^{3 / 2}
\end{aligned}
$$

the textbook result.

Similarly, the number density of particles in excited states due to thermal effects is given by

$$
\begin{aligned}
n_{\mathrm{thermal}}^{\mathrm{NR}} & =-\frac{1}{m} \frac{\partial}{\partial y} V_{\text {thermal }}^{\mathrm{NR}} \\
& =\frac{m^{3}}{\sqrt{2} \pi^{2}} \int_{0}^{\infty} \frac{d x x^{1 / / 2}(x+y-\delta)}{\left[(x+y-\delta)^{2}-\delta^{2} / 4\right]^{1 / 2}} \frac{1}{\exp \left\{\beta m\left[(x+y-\delta)^{2}-\delta^{2} / 4\right]^{1 / 2}\right\}-1} \\
& \rightarrow \frac{m^{3}}{\sqrt{2} \pi^{2}} \int_{0}^{\infty} \frac{d x x^{1 / 2}\left(x+y_{0}\right)}{\left(x^{2}+2 x y_{0}\right)^{1 / 2}} \frac{1}{\exp \left[\beta m\left(x^{2}+2 x y_{0}\right)^{1 / 2}\right]-1},
\end{aligned}
$$

where again we have used the tree-level expressions for $\delta$ and $y$ in the last line. At very low temperatures (high $\beta$ ) we can get an analytic expressions for $n_{\text {thermal }}^{\mathrm{NR}}$

$$
\begin{aligned}
\lim _{\beta m \sqrt{y_{0}} \rightarrow \infty} n_{\text {thermal }}^{\mathrm{NR}} & =\frac{y_{0}^{1 / 2} m^{3}}{2 \pi^{2}} \int_{0}^{\infty} \frac{1}{\exp \left(\beta m \sqrt{2 x y_{0}}\right)-1} \\
& =\frac{m^{2} T^{2}}{24 \sqrt{\pi} \sqrt{n a}} .
\end{aligned}
$$

As the temperature increases, the number of particles in excited states increases until at a critical temperaure $T_{c}$, all the charge can be stored in excited modes, and the symmetry is restored.

We can also use the effective potential to compute the ground-state energy of the Bose gas. From the definition of the partition function, we see that

$$
E \equiv\langle H\rangle=\left.\Omega \frac{\partial}{\partial \beta}\left(\beta V_{\text {eff }}\right)\right|_{\beta \mu \text { fixed }}
$$

or in the nonrelativistic limit the energy density is

$$
\rho \equiv \frac{E}{\Omega}=\left.\frac{\partial}{\partial \beta}\left[\beta V_{\mathrm{eff}}^{\mathrm{NR}}\right]\right|_{\beta y \text { fixed }} .
$$

The ground-state energy density $\rho_{0}$ is evaluated at $T=0$ when the thermal terms do not contribute, so using Eq. (5.12), we find

$$
\rho_{0}=\frac{m^{3} \delta^{2}}{32 \pi a}+\frac{m^{4}}{2 \sqrt{2} \pi^{2}} \int_{0}^{\infty} d x x^{1 / 2}\left(\frac{(x+\delta-y)(x+\delta)-\delta^{2} / 4}{\sqrt{(x+\delta-y)^{2}-\delta^{2} / 4}}-x-\delta+\frac{\delta^{2}}{8 x}\right),
$$

where we have dropped the rest mass contribution, $n m$. As written, $\rho_{0}$ is a function of $(\delta, y)$. To get an expression for the energy density in terms of the physical parameters, the number density and the scattering length, we must solve the equations $\left(\partial V_{\mathrm{eff}}^{\mathrm{NR}} / \partial y=0 ; \partial V_{\mathrm{eff}}^{\mathrm{NR}} / \partial \delta=0\right)$ which determine $(y, \delta)$ in terms of $(n, a)$. We have already done this at lowest 
order in Eq. (5.4). We can use this lowest-order solution in the second term on the right-hand side in the expression for the energy density, since this is already a one-loop correction. (The actual expansion parameter will emerge shortly.) However, when we substitute for $\delta$ in the first term, we must go beyond the zero-order solution. To do this, consider the equation $\partial V_{\mathrm{eff}}^{\mathrm{NR}} / \partial y=0$. This gives

$$
0=m n-\frac{\delta m^{3}}{8 \pi a}+\frac{m^{4}}{2 \sqrt{2} \pi^{2}} \int_{0}^{\infty} d x x^{1 / 2}\left(1-\frac{x+\delta-y}{\left[(x+\delta-y)^{2}-\delta / 4\right]^{1 / 2}}\right)
$$

which leads to

$$
\delta=\frac{8 \pi n a}{m^{2}}\left[1+\frac{m^{2}}{2 \sqrt{2} \pi^{2} n} \int_{0}^{\infty} d x x^{1 / 2}\left[1-\frac{x+\delta-y}{\left[(x+\delta-y)^{2}-\delta / 4\right]^{1 / 2}}\right]\right] .
$$

The first term is the tree-level term $\delta_{0}$, while the second is the one-loop correction. Again everywhere in the one-loop correction, it is consistent to set $\delta \rightarrow \delta_{0}, y \rightarrow y_{0}$. Then, the integral is elementary and we find

$$
\delta=\frac{8 \pi n a}{m^{2}}\left[1-\frac{8}{3 \sqrt{\pi}}\left(n a^{3}\right)^{1 / 2}\right] .
$$

We can use this expression for $\delta$ in the first term in Eq. (5.19) so that to this order the energy density is

$$
\begin{aligned}
\rho_{0} & =\frac{m^{3}}{32 \pi a}\left[\frac{8 \pi n a}{m^{2}}\left[1-\frac{8}{3 \sqrt{\pi}}\left(n a^{3}\right)^{1 / 2}\right]\right]^{2}+\frac{m^{4}}{2 \sqrt{2} \pi^{2}} \int_{0}^{\infty} d x x^{1 / 2}\left[\frac{\left(x+y_{0}\right)\left(x+2 y_{0}\right)-y_{0}^{2}}{\left(x^{2}+2 x y_{0}\right)^{1 / 2}}-x-2 y_{0}+\frac{y_{0}^{2}}{2 x}\right] \\
& =\frac{2 \pi n^{2} a}{m}\left[1+\frac{128}{15 \sqrt{\pi}}\left(n a^{3}\right)^{1 / 2}+\cdots\right] .
\end{aligned}
$$

The expansion parameter is therefore $\left(n a^{3}\right)^{1 / 2}$. This expression for the ground-state energy density agrees with the results of Lee and Yang [9] who used the binary collision method and with those of Lee, Huang, and Yang [10] using the pseudopotential method. Wu [11] took their calculations a step further and, using the pseudopotential method for a hard-sphere gas, found the next correction: $8(4 \pi / 3-\sqrt{3})\left(n a^{3}\right) \ln \left(12 \pi n a^{3}\right)$. It is an open question as to whether a consistent higher-order calculation (including two-loop effects) in $\phi^{4}$ theory would give an equivalent correction. As opposed to the approach of Ref. [7] though, it appears necessary to first calculate the effective potential and then take the nonrelativistic limit as we have done here.

Finally, by treating the effective potential to the same order in $\left(n a^{3}\right)$ as we did the energy, one can show that $V_{\mathrm{eff}}^{\mathrm{NR}}=\rho_{0}$. (The calculation is actually a bit simpler here since the first derivatives of $V_{\text {eff }}$ with respect to $y$ and $\delta$ vanish. Therefore, it is consistent to insert the tree-level values of $y$ and $\delta$ throughout.) Since the entropy is proportional to the difference between the energy (at zero temperature, $\left.E=\Omega \rho_{0}\right)$ and the free energy $\left(\Omega V_{\mathrm{eff}}^{\mathrm{NR}}\right)$, we see that at zero temperature the entropy vanishes. In fact, as one might expect this is also true of the relativistic expression provided we set the additive constant in Eq. (3.13) equal to zero, which we must do to conform to the third law, the Nernst theorem.

\section{TAKING THE HIGH-TEMPERATURE LIMIT}

The nonrelativistic limit discussed above provides valuable insight into our expression (3.13) for the one-loop effective potential. Much of the appeal of the effective potential formalism in quantum field theory, however, lies in its connection to phase structure-structure that often appears in high temperature, symmetry-changing phase transitions. To probe this structure we examine the high-temperature limit of Eq. (3.13).

Before proceeding to a limit-dependent discussion, however, we wish to make some observations about the exact expression (3.13) for $V_{\text {eff }}$.

First, we examine the one-loop integrals for $V_{\text {zero point }}$ and $V_{\text {thermal }}$ more carefully. Note that the zero-point contribution [from Eq. (3.16)] has no explicit temperature dependence. For technical reasons explained below, at high temperatures this term will become mildly temperature dependent, due to mass renormalization. However, the induced temperature dependence in this regime remains $\lambda$ suppressed relative to contributions of the other one-loop term, $V_{\text {thermal }}$. Thus we will neglect $V_{\text {zero point }}$ in our discussions of the high-temperature limit below.

Second, we consider $V_{\text {thermal }}$, Eq. (3.15), more closely. Note that the integrand (with weight $k^{2}$ ) vanishes in both the $k \rightarrow 0$ and $k \rightarrow \infty$ limits. It is maximized when $E_{ \pm}$ and $k$ are both comparable to the temperature. This is as expected for a thermal distribution of excitations: those excitations with physical energy $E_{ \pm} \sim T$ predominate.

'Finally, as with any perturbative calculation, the result (3.13) for $V_{\text {eff }}$ remains valid only when perturbation theory converges. Thus we must avoid the infrared divergences that plague theories near the critical pointthat is, naively, we must restrict ourselves to values of $\bar{\phi}$ and $T$ with

$$
\frac{\lambda \bar{\phi}^{2}}{m^{2}-\mu^{2}}<<1
$$

However, we can loosen this convergence constraint at 
higher temperature. We just established that modes with $k \sim T$ make the dominant contributions to $V_{\text {thermal }}$ (and hence, at high temperature, $V_{\text {eff }}$ ). Thus, at sufficiently high temperature, our integral (3.15) does not sample the infrared region, but instead a higher $k$ region with convergence factor

$$
\frac{\delta \bar{\phi}^{2}}{k^{2}+m^{2}-\mu^{2}} \sim \frac{\lambda \bar{\phi}^{2}}{T^{2}} .
$$

In any case, we must obey a convergence constraint. And, since we must obey it, we may as well exploit the constraint to simplify our expressions for the dispersion relations $E_{ \pm}$. We define, for convenience, the quantity

$$
E^{2}=|\mathbf{k}|^{2}+\widetilde{m}^{2},
$$

with $\widetilde{m}^{2}$ as defined in Eq. (3.21), and Taylor expand Eq. (3.9) in terms of the small quantity $\lambda \bar{\phi}^{2} /\left(E^{2}-\mu^{2}\right)$. We obtain

$$
\gamma=E^{2}-\mu^{2}-\frac{\lambda^{2} \bar{\phi}^{4}}{72\left(E^{2}-\mu^{2}\right)},
$$

giving, after an additional Taylor expansion in Eq. (3.17),

$$
\begin{aligned}
E_{ \pm} & =E-\frac{\lambda^{2} \bar{\phi}^{4}}{288 E\left(E^{2}-\mu^{2}\right)} \pm\left[\mu^{2}+\frac{\lambda^{2} \bar{\phi}^{4}}{144\left(E^{2}-\mu^{2}\right)}\right]^{1 / 2} . \\
& \equiv \widetilde{E} \pm \Delta .
\end{aligned}
$$

To proceed with the high-temperature limit, we consider two regimes. In the first, and more interesting, the energy differential $\Delta$ is dominated by $\mu$. This situation, which occurs for any non-negligible charge density, is where charge accommodation plays a significant role, as the capacity of modes to accommodate charge dominates their self-interactions. In the second regime, $\mu$ is small compared to self-interaction terms. This occurs only for perversely small initial charge densities, and we present it only because this regime, and not the charge-dominated one, contains the $\mu \rightarrow 0$ (or $n \rightarrow 0$ ) limit. We find, ultimately, that despite their existence in entirely different realms of analysis, both regimes display the same hightemperature behavior.

\section{A. The charge-dominated regime}

We now consider the integrand $j(k)$ of the one-loop thermal contribution displayed in Eq. (3.15):

$$
j(k) \equiv \ln \left(1-e^{-\beta(\tilde{E}+\Delta)}\right)\left(1-e^{-\beta(\tilde{E}-\Delta)}\right) .
$$

Because $\widetilde{E}$ is extremely close to $E$, whose functional dependence on $|\mathbf{k}|$ is quite simple, we Taylor expand $j(k)$ in $\widetilde{E}-E$ to get

$$
\begin{aligned}
j(k)= & \ln \left(1-e^{-\beta(E+\Delta)}\right)\left(1-e^{-\beta(E-\Delta)}\right) \\
& -\frac{\beta \lambda^{2} \bar{\phi}^{4}}{288 E\left(E^{2}-\mu^{2}\right)}\left(\frac{1}{e^{\beta(E+\Delta)}-1}+\frac{1}{e^{\beta(E-\Delta)}-1}\right) .
\end{aligned}
$$

Note that a power of $\beta$ enters with each power $\widetilde{E}-E$ of the Taylor expansion. Thus this technique helps us only in the relativistic limit we study here.

In the charge-dominated regime, $\Delta$ is dominated by $\mu$, and Taylor expanding Eq. (6.5) gives

$$
\Delta=\mu+\frac{\lambda^{2} \bar{\phi}^{4}}{288 \mu\left(E^{2}-\mu^{2}\right)} .
$$

We then Taylor expand $j(k)$ around $\Delta=\mu$ to give

$$
\begin{aligned}
j(k)= & \ln \left(1-e^{-\beta(E+\mu)}\right)\left(1-e^{-\beta(E-\mu)}\right) \\
& +\frac{\beta \lambda^{2} \bar{\phi}^{4}}{288 \mu E}\left(\frac{1}{E+\mu} \frac{1}{e^{\beta(E+\mu)}-1}-\frac{1}{E-\mu} \frac{1}{e^{\beta(E-\mu)} 1}\right) \\
& +O\left(\beta^{2}(\Delta-\mu)(\widetilde{E}-E)\right) .
\end{aligned}
$$

Plugging this expression back into Eq. (3.15) and recalling Eq. (6.5), we obtain the following expression for $V_{\text {thermal }}$ :

$$
V_{\text {thermal }}=\frac{1}{\beta} \frac{d^{3} k}{(2 \pi)^{3}} \ln \left(1-e^{-\beta(E+\mu)}\right)\left(1-e^{-\beta(E-\mu)}\right)+\int \frac{d^{3} k}{(2 \pi)^{3}} \frac{\lambda^{2} \bar{\phi}^{4}}{288 \mu E}\left[\frac{1}{E+\mu} \frac{1}{e^{\beta(E+\mu)}-1}-\frac{1}{E-\mu} \frac{1}{e^{\beta(E-\mu)}-1}\right]
$$

The first line in Eq. (6.10) resembles the contribution of a free theory, with fixed charge, except for the inclusion of the term $\lambda \bar{\phi}^{2} / 3$ in $E^{2}$ [through $\widetilde{m}^{2}$, see Eq. (3.21)]. Much of Haber and Weldon's analysis for the free theory extends simply to this expression, in particular their discussion of high-temperature limits. By rewriting the expression, which they call $J$ in terms of a recursive function

$$
J=-\frac{4}{\pi^{2} \beta^{4}} H_{5}(\beta \widetilde{m}, \mu / \widetilde{m}),
$$

they show $J$ to have the high-temperature limit

$$
\begin{aligned}
J= & -\frac{-\pi^{2} T^{4}}{45}+\frac{T^{2} \widetilde{m}^{2}}{12}-\frac{T^{2} \mu^{2}}{6} \\
& +\frac{T\left(\widetilde{m}^{2}-\mu^{2}\right)^{3 / 2}}{6 \pi}+O(\ln T) .
\end{aligned}
$$

The only difference from their result is the shift $m^{2} \rightarrow \widetilde{m}^{2}$. Its dominant effect is to generate the wellunderstood mass renormalization at higher temperature:

$$
m^{2} \rightarrow m^{2}+\frac{\lambda T^{2}}{18} .
$$


At this point a technical discussion is warranted. As Weinberg points out, the appearance of a quadratic mass correction at high temperature signals the breakdown of perturbation theory-for one-loop mass insertions become comparable to tree-level terms $[12,13]$. This can be addressed via one of two strategies. Weinberg chooses to renormalize the mass as in Eq. (6.13) at the tree level, then neglect one-loop corrections as perturbatively small. Fendley, instead, leaves the tree-level term unchanged, but sums over all mass insertions on graphs that contribute to the one-loop effective potential, thus restoring convergence of the loop expansion. This is accomplished by simply using the renormalized mass (6.13) in place of the mass in expressions obtained for the one-loop effective potential $[12,14]$. We follow Fendley's approach here, as we are interested in the $\mu$ and $T$ dependence of the oneloop corrections. Technically, then, the $m^{2}$ and $\widetilde{m}^{2}$ that appear in $V_{\text {zero point }}$ and $V_{\text {thermal }}$ above should be corrected by the additive term $\lambda T^{2} / 18$. Derivation of Haber and Weldon's high-temperature limit (6.12) goes through as before (with $\widetilde{m}^{2} \rightarrow \widetilde{m}^{2}+\lambda T^{2} / 18$ ), as the dimensionless quantity $\beta^{2} \widetilde{m}^{2}$ remains dimensionless. Furthermore, all additive terms induced into Eq. (6.12) by the shift end up being a factor of $\lambda$ smaller than preexisting terms. Terms induced by shifting $\widetilde{m}^{2}$ in $V_{\text {zero point }}$ are also $\lambda$ suppressed relative to terms we retain in expression (6.12) for $V_{\text {thermal }}$. So, while we acknowledge use of the temperature-dependent mass for one-loop calculations, we do so for pedantic reasons only, to ensure that, for example, two-loop corrections are not comparable to the precision we quote in our one-loop result. Pragmatically, however, the difference in calculated results appears negligible.

We now proceed to the second line in expression (6.10) for $V_{\text {thermal }}$. To evaluate it, we must find the hightempeature limit to the integral

$I \equiv \int \frac{d^{3} k}{(2 \pi)^{3}} \frac{1}{E}\left(\frac{1}{E+\mu} \frac{1}{e^{\beta(E+\mu)}-1}-\frac{1}{E-\mu} \frac{1}{e^{\beta(E-\mu)}-1}\right)$.

Because it depends on the variables $\beta, \mu$, and $\widetilde{m}^{2}$ (implicitly through $E$ ), $I$ can be related to the free thermal contribution $J$ :

$$
\partial_{\beta} I=\frac{2}{\beta} \partial_{(\tilde{m}, 2)} \partial_{\mu} J
$$

Using the Haber and Weldon high-temperature limit (6.12) for $J$ then gives

$$
I=\frac{\mu T}{2 \pi \sqrt{\tilde{m}^{2}-\mu^{2}}} .
$$

Combining these results, Eqs. (6.12) and (6.16), yields the high-temperature limit for $V_{\text {thermal }}$ in the chargedominated regime. We retain only terms linear in $T$ or higher, and only the dominant portions of their coefficients (that is, we neglect $\lambda$-suppressed contributions). In no place, however, do we neglect leading $\mu, \bar{\phi}$, or $T$ dependence. We obtain then

$$
\begin{aligned}
V_{\text {thermal }}= & \frac{-\pi T^{4}}{45}-\frac{T^{2} \widetilde{m}^{2}}{12}-\frac{T^{2} \mu^{2}}{6} \\
& +\frac{T\left(\widetilde{m}^{2}-\mu^{2}\right)^{3 / 2}}{6 \pi}+\frac{T \lambda^{2} \bar{\phi}^{4}}{576 \pi \sqrt{\tilde{m}^{2}-\mu^{2}}} .
\end{aligned}
$$

Note that the leading behavior of $\bar{\phi}, \mu$, and $T$ is all determined by terms of order $T^{2}$ or higher. These terms are unchanged from the free case, with the replacement $m^{2} \rightarrow \widetilde{m}^{2}$. This makes further analysis of thermodynamic quantities and their evolution particularly simple.

\section{B. The interaction-dominated regime}

In the interaction-dominated regime, the energy shift $\Delta$ [from Eq. (6.5)] is dominated throughout the range of integration, by the term $\lambda \bar{\phi}^{2} / 12 \sqrt{E^{2}-\mu^{2}}$. This occurs only for pathologically small values of $\mu$, although these admittedly are the values of interest in the $n \rightarrow 0$ limit. We see this as follows.

First, it is simple to overestimate the upper bound on $\mu$ within this regime. For $\Delta^{2}$ to be dominated by the term $\lambda^{2} \bar{\phi}^{4} / 144\left(E^{2}-\mu^{2}\right)$, the quantity

$$
R(k)=\frac{\lambda^{2} \bar{\phi}^{4}}{\mu^{2}\left(E^{2}-\mu^{2}\right)}
$$

must be large. $R$ is maximal at $k=0$ :

$$
\begin{aligned}
R(k=0) & \leq \frac{\lambda^{2} \bar{\phi}^{4}}{\mu^{2}\left(m^{2}-\mu^{2}\right)} \\
& =\frac{m^{2}-\mu^{2}}{\mu^{2}}\left(\frac{\lambda \bar{\phi}^{2}}{m^{2}-\mu^{2}}\right)^{2} .
\end{aligned}
$$

We have established that $\lambda \bar{\phi}^{2} /\left(m^{2}-\mu^{2}\right)$ is small within the realm of perturbation theory, so $\mu^{2} / m^{2}$ must be $e x-$ tremely small, even in this overestimate:

$$
\frac{\mu^{2}}{m^{2}} \ll\left(\frac{\lambda \bar{\phi}^{2}}{m^{2}}\right)^{2}
$$

At high temperature, this upper bound becomes even more stringent. Because the thermal fluctuations are dominated by modes with $E \sim T$ (as discussed at the end of Sec. V), the "large" quantity $R$ becomes

$$
R(k \sim T)=\frac{\lambda^{2} \bar{\phi}^{4}}{\mu^{2} T^{2}} .
$$

This further constrains $\mu$ :

$$
\frac{\mu^{2}}{m^{2}} \ll\left(\frac{\lambda \bar{\phi}^{2}}{m^{2}}\right)^{2}\left(\frac{m^{2}}{T^{2}}\right) .
$$

Note that this restriction eliminates all physically significant values of $\mu$ (or $n$, as seen in Sec. VII below). This regime thus has only formal relevance, and only because we must pass through it in taking any $\mu \rightarrow 0$ limit.

With these disclaimers about its extremely limited scope established, we now present the interactiondominated limit. Here we may Taylor expand $\Delta$ as follows: 


$$
\begin{aligned}
\Delta & =\frac{\lambda \bar{\phi}^{2}}{12 \sqrt{E^{2}-\mu^{2}}}\left[1+\frac{72 \mu^{2}\left(E^{2}-\mu^{2}\right)}{\lambda^{2} \bar{\phi}^{4}}\right) \\
& =\frac{\lambda \bar{\phi}^{2}}{12 E}\left[1+\frac{72 \mu^{2} E^{2}}{\lambda^{2} \bar{\phi}^{4}}\right)
\end{aligned}
$$

where we have neglected terms with relative suppression factors $\mu^{2} / E^{2},\left(\lambda \bar{\phi}^{2} / E^{2}\right)^{2}$. Note that $\Delta$ is much smaller than $E$, but much larger than the energy shift $\widetilde{E}-E$ [cf. Eq. (6.5)].

In this case it makes sense to Taylor expand the $j(k)$ of
Eq. (6.6) about the values $\widetilde{E} \pm \Delta=E$. For consistency, we must Taylor expand to second order, as the larger differentials $\pm \Delta$ cancel at first order. We obtain

$$
\begin{aligned}
j(k)= & 2 \ln \left(1-e^{-\beta E}\right)-\frac{\beta \lambda^{2} \bar{\phi}^{4}}{144 E^{3}} \frac{1}{e^{\beta E}-1} \\
& -\frac{\beta^{2} \lambda^{2} \bar{\phi}^{4}}{144 E^{4}}\left(1+\frac{144 \mu^{2} E^{2}}{\lambda^{2} \bar{\phi}^{4}}\right) \frac{e^{\beta E}}{\left(e^{\beta E}-1\right)^{2}},
\end{aligned}
$$

again neglecting relatively suppressed terms. Plugging this expression back into Eq. (3.15). gives the following result for $V_{\text {thermal }}$ :

$$
V_{\text {thermal }}=\int \frac{d^{3} k}{(2 \pi)^{3}} \frac{2}{\beta} \ln \left(1-e^{-\beta E}\right)-\beta \mu^{2} \frac{e^{\beta E}}{\left(e^{\beta E}-1\right)^{2}}-\frac{\lambda^{2} \bar{\phi}^{4}}{144} \int \frac{d^{3} k}{(2 \pi)^{3}}\left(\frac{1}{E^{3}} \frac{1}{e^{\beta E}-1}+\frac{\beta}{E^{2}} \frac{e^{\beta E}}{\left(e^{\beta E}-1\right)^{2}}\right)
$$

The first term in Eq. (6.25) consists of the thermal contribution of a free theory with $\mu=0$ and $m^{2}$ shifted to $\widetilde{m}^{2}$. Its high-temperature behavior is therefore given by the shifted Haber and Weldon limit (6.12), with $\mu$ set to zero:

$J(\mu=0)=\frac{-\pi^{2} T^{4}}{45}+\frac{T^{2} \widetilde{m}^{2}}{12}+\frac{T \widetilde{m}^{3}}{6 \pi}+O(\ln T)$.

To evaluate the remaining terms we must perform the integrations

$$
I_{1} \equiv \int \frac{d^{3} k}{(2 \pi)^{3}}\left(\frac{1}{E^{3}} \frac{1}{e^{\beta E}-1}+\frac{\beta}{E^{2}} \frac{e^{\beta E}}{\left(e^{\beta E}-1\right)^{2}}\right)
$$

and

$$
I_{2} \equiv \int \frac{d^{3} k}{(2 \pi)^{3}} \frac{e^{\beta E}}{\left(e^{\beta E}-1\right)^{2}} .
$$

As in the charge-dominated case, we exploit their relationships with the variables $\beta$ and $\widetilde{m}^{2}$ (through $E$ ) to relate $I_{1}$ and $I_{2}$ to $J(\mu=0)$ :

$$
\begin{aligned}
& I_{1}=-2 \frac{\partial^{2} J}{\partial\left(\widetilde{m}^{2}\right)^{2}}, \\
& I_{2}=-\frac{\partial^{2} J}{\partial \beta \partial\left(\widetilde{m}^{2}\right)} .
\end{aligned}
$$

This result, along with Eq. (6.26), gives us the following high-temperature limit to our expression (6.25) for $V_{\text {thermal }}$ in the interaction-dominated regime:

$$
\begin{aligned}
V_{\text {thermal }}= & \frac{-\pi^{2} T^{4}}{45}+\frac{T^{2} \widetilde{m}^{2}}{12}+\frac{T \widetilde{m}^{3}}{6 \pi}+\frac{T \lambda 2^{2} \bar{\phi}^{4}}{576 \pi \tilde{m}} \\
& -\frac{\mu^{2} T^{2}}{6}-\frac{\mu^{2} \tilde{m} T}{4 \pi} .
\end{aligned}
$$

Note that this expression coincides with the small $\mu^{2} / \widetilde{m}^{2}$ limit of our result (6.17) in the charge-dominated regime. Again the leading behavior of $\bar{\phi}, \mu$, and $T$ are determined by terms of order $T^{2}$ or higher. And these terms again correspond to the free case, with $m^{2}$ replaced by $\widetilde{m}^{2}$. Thus the analysis of the charge-dominated and interaction-dominated regimes collapse to the same re- sult, although obtained by entirely incompatible Taylor expansions. The simple form of this result facilitates further analysis of thermodynamic quantities and their evolution.

\section{VACUUM STRUCTURE AND CHARGE ACCOMMODATION}

As mentioned in Sec. II, information about a theory's phase structure and charge distribution are encoded in the $\bar{\phi}$ and $\mu$ dependence of the effective potential. And from Sec. VI A, the high-temperature effective potential for an $\mathrm{SO}(2)$-symmetric theory has the form, to one loop,

$$
\begin{aligned}
V_{\mathrm{eff}}= & V_{\text {tree }}+V_{\text {thermal }} \\
= & \frac{1}{2}\left(m^{2}-\mu^{2}\right) \bar{\phi}^{2}+\frac{\lambda}{24} \bar{\phi}^{4}+\mu \mathrm{n} \\
& -\frac{\pi^{2} T^{4}}{45}+\frac{T^{2} \widetilde{m}^{2}}{12}-\frac{\mu^{2} T^{2}}{6},
\end{aligned}
$$

where we have used Eq. (3.14) for $V_{\text {tree }}$ with physical renormalization conditions, $n$ the charge density $\bar{Q} / \Omega$ and $\widetilde{m}^{2}$, the shifted mass, given by Eq. (3.21). Note that we have dropped all subleading $\mu, T$, and $\bar{\phi}$ dependences. We now explore the qualitative implications this expression for $V_{\text {eff }}$ contains about the theory's phase structure and its capacity for accommodating charge.

Phase structure depends on the minima of the effective potential. Remembering that $\bar{\phi}$ appears in $V_{\text {eff }}$ both explicitly and implicitly, through $\widetilde{m}^{2}$, we obtain

$$
\frac{\partial V_{\mathrm{eff}}}{\partial \bar{\phi}}=\left[m^{2}-\mu^{2}+\frac{\lambda \bar{\phi}^{2}}{6}+\frac{\lambda T^{2}}{18}\right] \bar{\phi} \text {. }
$$

Thus $V_{\text {eff }}$ has two minima: $\bar{\phi}=0$, with unbroken symmetry, and

$$
\begin{aligned}
\bar{\phi}^{2} & =\frac{6}{\lambda}\left[\mu^{2}-m^{2}-\frac{\lambda T^{2}}{18}\right] \\
& =\frac{6}{\lambda}\left[\mu^{2}-m^{2}(T)\right],
\end{aligned}
$$


with broken symmetry. The phase transition occurs when $\bar{\phi}^{2}$ can first become non-negative, at

$$
T_{c}^{2}=\frac{18\left[\mu^{2}\left(T_{c}\right)-m^{2}\right]}{\lambda}
$$

In general, $\mu$ has temperature dependence as dictated by the charge-conservation constraint. However, many important qualitative points can be extracted from the simple case of constant $\mu$ (which does arise in some physical settings, as shown in Sec. VIII). Here we have lowtemperature symmetry breaking, with

$$
\bar{\phi}^{2}=\frac{1}{3}\left(T_{c}^{2}-T^{2}\right)
$$

below $T_{c}$. This of course requires that $T_{c}^{2}$ be positive, i.e., that $m^{2}<\mu^{2}$, replacing the usual condition $m^{2}<0$ for low-temperature symmetry breaking. We check the consistency of this low-temperature symmetry-breaking scenario by considering the mass,

$$
\begin{aligned}
\frac{\partial^{2} V_{\mathrm{eff}}}{\partial \bar{\phi}^{2}} & =m^{2}(T)-\mu^{2}+\frac{\lambda \bar{\phi}^{2}}{2} \\
& = \begin{cases}\frac{\lambda}{18}\left(T^{2}-T_{c}^{2}\right) & \text { for unbroken symmetry, } \\
\frac{\lambda}{9}\left(T_{c}^{2}-T^{2}\right) & \text { for broken symmetry, }\end{cases}
\end{aligned}
$$

which shows the unbroken state is indeed minimal above $T_{c}$, with the broken state minimal below $T_{c}$.

Note that the nonzero vacuum expectation value (VEV) $\bar{\phi}$, as defined in Eq. (7.3), occurs in a regime where perturbation theory is beset by infrared divergences (cf. the discussion at the end of Sec. V). We can evade this trouble in two ways. First, as also discussed in Sec. VI A, at high temperature the dominant contributions to $V_{\text {thermal }}$ come from states with $k \sim T$. Thus, at sufficiently high temperature, we simply do not sample the infrared region and need not worry about preventing infrared divergences.

A second resolution is to add uncharged heavy fields to the theory, which induce effective nonrenormalizable $\bar{\phi}$ interactions that shift the VEV:

$$
\bar{\phi}^{2} \rightarrow \frac{6}{\lambda}\left[\mu^{2}-m^{2}(T)-a \bar{\phi}^{4}\right] .
$$

This possibility may be interesting in its own right - for example, Benson and Widrow use it, with negative $\lambda$, to obtain high-temperature symmetry breaking [12]. Note that while this addition changes the VEV, it does not affect the critical temperature, when $\bar{\phi}^{2}$ first becomes non-negative. That remains

$$
T_{c}^{2}=\frac{18\left[\mu\left(T_{c}\right)-m^{2}\right]}{\lambda} .
$$

For high-temperature symmetry breaking, then, $\lambda$ is negative and $m^{2}>\mu^{2}$. Above $T_{c}, \phi$ condenses, below $T_{c}$, symmetry is restored.

It is interesting to note that in both cases, lowtemperature symmetry breaking and the more contrived high-temperature symmetry breaking, the presence of a nonzero charge acts to enhance symmetry breaking. This can be seen most naively by examining its effect on the critical temperature. For low-temperature symmetry breaking, in the absence of $\mu$, the critical temperature is the point at which the renormalized mass vanishes:

$$
T_{c}^{2}=18\left|m^{2}\right| / \lambda \text {. }
$$

With nonzero $\mu$ this critical temperature rises to the value (7.4), i.e.,

$$
T_{c}^{2} \rightarrow 18\left[\left|m^{2}\right|+\mu^{2}\left(T_{c}\right)\right] / \lambda .
$$

Thus, as the system cools, the symmetry breaks earlier than it would in a sector of zero charge. Similarly, for high-temperature symmetry breaking in the absence of $\mu$,

$$
T_{c}^{2}=18 m^{2} /|\lambda| \text {. }
$$

With nonzero $\mu$ the critical temperature falls to Eq. (7.9),

$$
T_{c}^{2}=18\left[m^{2}-\mu^{2}\left(T_{c}\right)\right]|\lambda|,
$$

so the symmetry remains broken longer than it would in the absence of charge.

This enhancement makes sense given the conventional interpretation of phase transitions as Bose condensation. This contends that whenever charge cannot be fully accommodated by thermally excited states, $\phi$ must condense so that charge may occupy the zero-momentum phase excitations of $\bar{\phi}$, i.e., the Goldstone modes. We investigate this by examining the charge distribution $n$.

Recall, from Secs. II and III, the relation between $n$ and the $\mu$ follows from minimizing the effective potential with respect to $\mu$ :

$$
n=\mu \bar{\phi}^{2}-\frac{\partial V_{\text {thermal }}}{\partial \mu},
$$

where we have neglected the zero-point contribution. Also note that the effective potential's leading hightemperature $\mu$ dependence is unchanged from the result of Haber and Weldon for a free theory; the shift $m^{2} \rightarrow \widetilde{m}^{2}$ in calculating thermal contributions does not affect it. Thus the thermal contribution is simply

$n_{\text {thermal }}=-\frac{\partial J}{\partial \mu}=\int \frac{d^{3} k}{(2 \pi)^{3}}\left(\frac{1}{e^{\beta(E-\mu)}-1}-\frac{1}{e^{\beta(E+\mu)}-1}\right)$,

where, $J$ the mass-shifted free expression, is given by the first line of Eq. (6.10).

Formally, then, we see the basis for the Haber-Weldon interpretation: Eq. (7.15) represents the charge density by thermal excitations for a Bose gas of particles and antiparticles. We also note their observation that the integral (7.15) excludes zero-momentum excitations, as the integrand has zero weight here. Thus the total charge density, given by also including the tree-level contribution

$$
n_{\text {tree }}=\mu \bar{\phi}^{2},
$$

is readily interpreted as a sum of (1) the charge density carried by a bath of thermal modes, and (2) that carried 
by the zero-momentum (Goldstone) modes. Had we not done our calculation with a chemical potential initially, we would have found the fields $\phi_{1}$ and $\phi_{2}$ to be rotating in time with frequency $\mu$. Thus, excitation of the Goldstone modes produces a time-dependent, rotating condensate:

$$
\begin{aligned}
& \bar{\phi}_{1} \rightarrow \cos \mu t \bar{\phi}_{1}+\sin \mu t \bar{\phi}_{2}, \\
& \bar{\phi}_{2} \rightarrow-\sin \mu t \bar{\phi}_{1}+\cos \mu t \bar{\phi}_{2},
\end{aligned}
$$

whose charge is given by the angular-momentum-like term $\mu \bar{\phi}^{2}$. In the high-temperature limit, using Eq. (7.1), the sum of these two contributions simply becomes

$$
n=\mu \bar{\phi}^{2}+\mu T^{2} / 3 \text {. }
$$

Thus the charge fraction stored in the Goldstone modes of a condensed state is given by

$$
x=\frac{\bar{\phi}^{2}}{\bar{\phi}^{2}+T^{2} / 3} \text {. }
$$

For our simple theory, with low-temperature symmetry breaking, Eq. (7.5) yields

$$
x=1-T^{2} / T_{c}^{2} \text { below } T_{c} .
$$

Effective theories with high-temperature symmetry breaking give different, model-dependent values for $x$. For any theory, whenever $x$ approaches unity, excited Goldstone modes vastly outnumber excited thermal modes in the theory. This means that the field $\phi$ is well approximated everywhere by the rotating VEV (i.e., the VEV plus its Goldstone excitations), as thermal fluctuations are not sufficient to wash out the tendency of $\phi$ to sit in its minimum. Thus what is often only a very gross measure of the temperature-dependent state, the VEV $\bar{\phi}$, becomes a reliable indicator of the microphysics of $\phi$. In this case, semiclassical analysis that follows the evolution of the field $\phi$ in its temperature-dependent effective potential $V_{\text {eff }}$ provides a valid description of the theory. Such analysis is employed by many practitioners of inflation theory, and is crucial to recent work of Benson and Widrow on dynamically enacting first-order phase transitions without tunneling [12].

\section{EVOLUTION OF THE CHARGED VACUUM}

There are two physically interesting settings in which our theory, with low-temperature symmetry breaking, may evolve. In the first, or cosmological, setting, the Universe expands at constant entropy $S$. Our expression for $V_{\text {eff }}$ gives the entropy density

$$
s=-\frac{\partial V_{\mathrm{eff}}}{\partial T}=\frac{4 \pi^{2} T^{3}}{45},
$$

so we must require that, keeping $n / s$ constant,

$$
n=\eta T^{3} \text {. }
$$

This is just the familiar requirement in an expanding Universe that the charge per comoving volume remain constant. For more terrestrial applications, we also consider a setting with constant volume $V$, where

$$
n=n_{0}
$$

itself remains constant.

Both of these settings have

$$
n=\mu T^{2} / 3
$$

in the unbroken phase. This is easily solved for manifest symmetry:

$$
\mu=\left\{\begin{array}{l}
3 \eta T \text { at constant } S, \\
3 n_{0} / T^{2} \text { at constant } V .
\end{array}\right.
$$

In the broken phase, we have

$$
n=\mu \bar{\phi}^{2}+\mu T^{2} / 3=\frac{6 \mu}{\lambda}\left(\mu^{2}-m^{2}\right)
$$

where we have used Eq. (7.18) and Eq. (7.3) for the VEV. Using Eq. (7.4) for $T_{c}^{2}$, we note that $\mu$ is continuous through the phase transition, as the cubic equation (8.6) gives

$$
n=\mu\left(T_{c}\right) T_{c}^{2} / 3
$$

at $T_{c}$ in accord with its value (8.4) from the other direction. In general, Eq. (8.6) for $\mu$ is hard to solve, but it simplifies in two limits: the low-density limit, with $\lambda n \ll m^{3}$, and the high-density limit, where $\lambda n \gg m^{3}$ [3].

\section{A. The low-density limit}

In the low-density limit, both $n$ and $\mu$ are small and have minimal impact on the evolution of the theory. Here, taking $m^{2}<0$ so the theory has low-temperature symmetry breaking in the absence of charge, Eq. (8.6) yields

$$
\mu=\frac{\lambda n}{6\left|m^{2}\right|} \ll|m| .
$$

By design, then, $\mu$ has little effect on $T_{c}$, so we expand about its value in the absence of $\mu$ :

$$
T_{c}^{2} \equiv \frac{18\left|m^{2}\right|}{\lambda}(1+\delta) \text {. }
$$

Setting Eq. (7.3) for $\bar{\phi}^{2}$ to zero at $T_{c}$ yields

$$
\delta=\mu^{2}\left(T_{c}\right) /\left|m^{2}\right|
$$

that is, the critical temperature is shifted slightly higher than its value in the absence of $n$, as expected. Finally we note that $\mu$ is continuous at the transition, though its derivatives are not. For constant volume, we obtain the solution

$$
\mu=\left\{\begin{array}{l}
3 n_{0} / T^{2} \text { above } T_{c} \\
\frac{\lambda n_{0}}{6 m^{2}}=3 n_{0} / T_{c}^{2} \text { below } T_{c}
\end{array} \text { at constant } V .\right.
$$

For isentropic expansion, we get instead 


$$
\mu=\left\{\begin{array}{lr}
3 \eta T \text { above } T_{c} & \text { at constant } S \\
\frac{\lambda \eta}{6 m^{2}} T^{3}=3 \eta T^{3} / T_{c}^{2} & \text { below } T_{c}
\end{array}\right.
$$

\section{B. The high-density limit}

In the high-density limit, both $n$ and $\mu$ are large and their effect on the evolution of the theory is extreme. Here Eq. (8.6) for $\mu$ yields

$$
\mu=\left(\frac{\lambda n}{6}\right)^{1 / 3} \gg m \text {. }
$$

This large value for $\mu$ has its mildest effect on constant volume evolution. Here Eq. (7.4) for $T_{c}$ becomes

$$
T_{c}^{2}=3\left(\frac{6 n_{0}^{2}}{\lambda}\right)^{1 / 3} \gg \frac{18\left|m^{2}\right|}{\lambda} .
$$

Thus symmetry breaking occurs much earlier, at a much higher temperature, than it would in the absence of $n$. We note that, as in the low-density limit for constant volume evolution, $\mu$ is continuous at the transition, though its derivatives are not:

$$
\mu=\left\{\begin{array}{l}
3 n_{0} / T^{2} \text { above } T_{c} \\
{\left[\frac{\lambda n_{0}}{6}\right]^{1 / 3}=3 n_{0} / T_{c}^{2} \text { below } T_{c}}
\end{array} \text { at constant } V .\right.
$$

Note that this is the same form obtained in the lowdensity limit, the only difference being a vast increase in $T_{c}$ at high density.

While reasonable for constant volume evolution, the high-density limit truly dominates parameter space for systems undergoing high-temperature expansion. It occurs whenever

$$
\lambda \eta \gg m^{3} / T^{3},
$$

and since the right-hand side is by assumption extremely small, this is virtually always. And in this natural setting of isentropic expansion, the large, high-density solution (8.13) for $\mu$ has its most dramatic impact. Here, Eq. (7.4) for $T_{c}$ gives

$$
T_{c}^{2}=\frac{18 m^{2}}{\lambda\left[\left(162 \eta^{2} / \lambda\right)-1\right]},
$$

since

$$
\mu\left(T_{c}\right)=3 \eta T_{c}
$$

by continuity from the unbroken phase. Note that we have no way of relating this value for $T_{c}^{2}$ to typical critical temperatures for uncharged sectors of the theory, as it depends on the arbitrary parameter

$$
\chi \equiv\left(\frac{162 \eta^{2}}{\lambda}\right) \text {. }
$$

Further note that

$$
\frac{\mu^{2}\left(T_{c}\right)}{m^{2}}=\frac{\chi}{\chi-1} ;
$$

that is, unless $\chi$ is very close to one, we cannot neglect the $m^{2}$ term in Eq. (8.6) to obtain the high-density limit (8.13) for $\mu$. In cases where the high-density limit does hold, however, either at temperatures far enough above $T_{c}$ that $\mu^{2}(T) \gg m^{2}$ or simply for $\chi$ close enough to one, near $T_{c}$, Eq. (7.3) gives the VEV in the broken phase:

$$
\bar{\phi}^{2}=\frac{1}{3}\left(\chi^{1 / 3}-1\right) T^{2}-\frac{6 m^{2}}{\lambda} \text {. }
$$

When this expression becomes non-negative, symmetry breaking occurs.

Thus our simple $\phi^{4}$ theory gives different behavior in four realms of parameter space. The first two correspond to negative $\mathrm{m}^{2}$, which, as discussed in the previous section, induces low-temperature symmetry breaking in the absence of charge. Such a theory has two behaviors in the high-density limit: for $\chi<1$, Eq. (8.21) for the VEV assumes positive values for low temperatures only. Thus we again get low-temperature symmetry breaking, with

$$
\bar{\phi}^{2}=\frac{1}{3}\left(|\chi-1| T_{c}^{2}-\left|\chi^{1 / 3}-1\right| T^{2}\right)
$$

below the modified $T_{c}$ of Eq. (8.17). For $\chi>1$, however, $\phi$ has the nonzero VEV

$$
\bar{\phi}^{2}=\frac{1}{3}\left(\chi^{1 / 3}-1\right) T^{2}+\frac{6\left|m^{2}\right|}{\lambda}
$$

at all temperatures, thus the symmetry is never restored. This change in the phase diagram of the theory is not an artifact of our high-density approximation: indeed, our approximation becomes increasingly good at high temperatures, where the anticipated symmetry restoration simply does not occur. We show in Fig. 1 a plot of the VEV $\bar{\phi}$ for specific parameter choices within these two regimes, computed numerically from the full Eq. (8.6) constraining $\mu$. This shows the two possible phase diagrams distinctly: for $\chi<1$ there is low-temperature symmetry breaking only; for $\chi>1$, the symmetry breaking persists at all temperatures.

Even theories with positive $\mathrm{m}^{2}$, which lack any phase structure in the absence of charge, can behave nontrivially in the high-density limit. We have the following possibilities: for $\chi<1$, the right-hand side of Eq. (8.21) for the VEV always remains negative; thus $\bar{\phi}$ must be zero and the symmetry remains unbroken always. For $\chi>1$, however, the right-hand side of Eq. (8.21) becomes positive at high temperatures, so $\bar{\phi} \neq 0$ is the preferred state. Thus high charge density induces high-temperature symmetry breaking, with

$$
\bar{\phi}^{2}=\frac{1}{3}\left[\left(\chi^{1 / 3}-1\right) T^{2}-(\chi-1) T_{c}^{2}\right]
$$

above the $T_{c}$ of Eq. (8.17). Plots of an exact numerical solution within this regime are shown in Fig. 2; they confirm both the high-temperature symmetry breaking as well as the asymptotically linear form of $\mu$ presented below. The validity of this symmetry breaking, as well as the symmetry breaking of the $m^{2}<0$ cases, is confirmed 


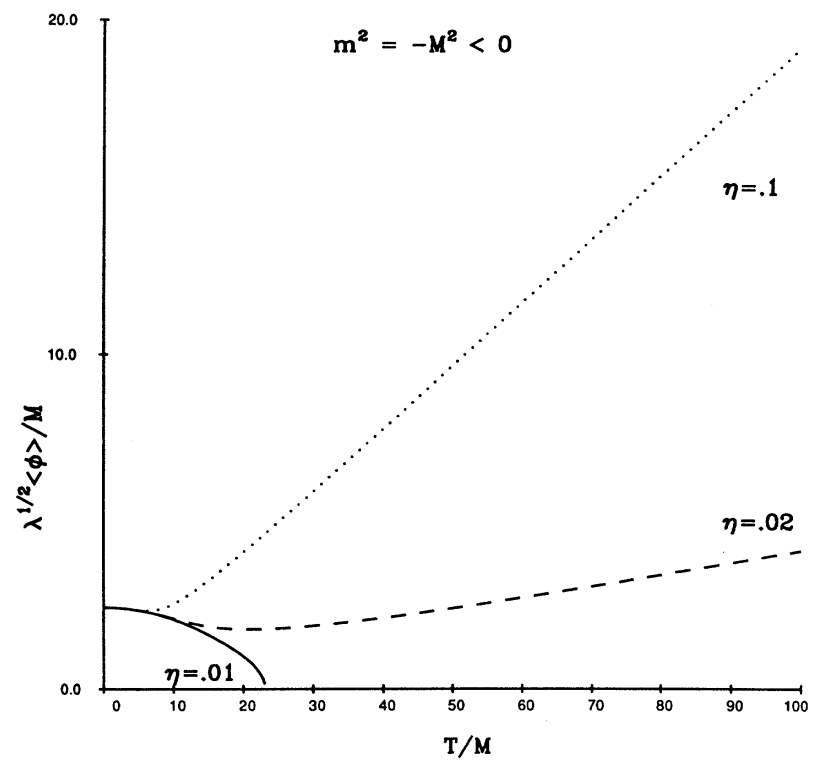

FIG. 1. The behavior of a $|\phi|^{4}$ theory with charge $n=\eta T^{3}$ and a negative mass squared. For small charge, the spontaneously broken symmetry gets restored at higher temperature (solid line with $\eta=0.01$ ). When the charge increases, though, so that the parameter $\chi$ defined in the the text is greater than one (dashed and dotted curves), the symmetry is never restored.

by examining the general mass formula $\partial^{2} V_{\text {eff }} / \partial \bar{\phi}^{2}$ of Eq. (7.6). It gives

$$
\frac{\partial^{2} V_{\text {eff }}}{\partial \bar{\phi}^{2}}=\frac{\lambda}{3} \bar{\phi}^{2}
$$

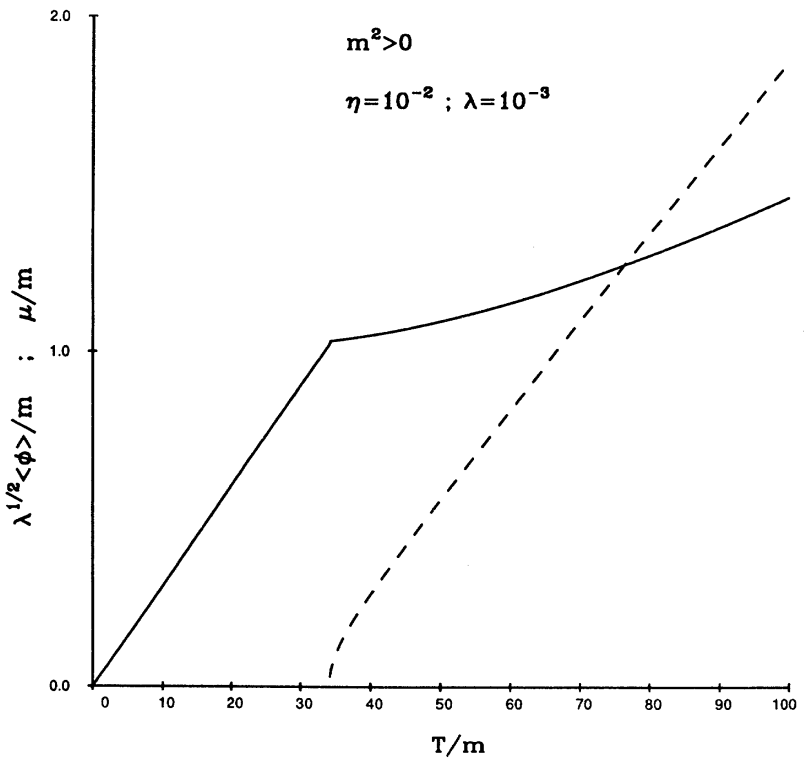

FIG. 2. The chemical potential (solid curve) and the expectation value of $\phi$ (dashed curve) vs temperature when the number density varies as $n=\eta T^{3}$. Note that this theory $\left(m^{2}>0\right)$ would ordinarily $(\eta=0)$ have no spontaneously broken phase. Because of the nonzero $\eta$, though, at high $T$ the charge cannot be stored in thermal modes. Here $\chi>1$.

for all the cases of broken symmetry discussed above (i.e., those phases with $\bar{\phi}^{2}>0$ ). Thus in these cases the effective potential is truly minimized in the broken state, and spontaneous symmetry breaking occurs.

Finally, we summarize these cases of high-density phase evolution during isentropic expansion:

$\mu=\left\{\begin{array}{cc}3 \eta T \text { for unbroken symmetry: when } m^{2}>0, \chi<1 ; m^{2}>0, \chi>1 \text { below } T_{c} ; \text { or } m^{2}<0, \chi<1 \text { above } T_{c} & \text { at constant } S \\ {\left[\frac{\lambda \eta}{2}\right]^{1 / 3} T=3 \eta \chi^{-1 / 3} T \text { for broken symmetry: }} & \text { when } m^{2}<0, \chi>1 ; m^{2}>0, \chi>1 \text { above } T_{c} ; \\ \text { or } m^{2}<0, \chi<1 \text { below } T_{c}\end{array}\right.$

Recall that the broken-symmetry solution is not necessarily valid near $T_{c}$, but holds only asymptotically at temperatures where $\mu^{2} \gg m^{2}$. Thus the discontinuity that appears for $\mu$ above should not be taken seriously. However, the striking change that high-charge density induces in high-temperature phase structure, either through the appearance of new high-temperature symmetry breaking or through the vanishing of anticipated high-temperature symmetry restoration, is an asymptotic and, hence, reliable, result.

\section{CONCLUSIONS}

We have calculated the exact solution for the finitetemperature effective potential, to one loop, in a selfinteracting theory with fixed $\mathrm{U}(1)$ charge. From the lowtemperature limit of this exact solution, we were able to extract many of the standard results of the nonrelativistic Bose gas. We also found the high-temperature limit and described the effects of nonzero charge on vacuum structure and critical temperatures. Pursuing the convention- 
al interpretation of phase transitions as Bose condensation, we investigated the distribution of charge between the thermal modes of the system and the Goldstone modes of a condensed VEV. We established a criterion for the validity of the semiclassical approximation, which holds when charge is concentrated in the Goldstone modes. Finally we presented a full solution of the phase evolution of the theory in two reasonable limits. showing that nonzero charge can dramatically accelerate a symmetry-breaking phase transition or even alter the number of phases appearing in the evolution of the system.

\section{ACKNOWLEDGMENTS}

We thank Lowell Brown, Sidney Coleman, Brian Greene, Larry Widrow, and T. T. Wu for useful discussions. S.D. is pleased to acknowledge the influence of courses by Alan Guth and David Nelson. J.B. would like to thank John Ellis for the hospitality of the Theory Division at CERN where some of this work was carried out. K.B. was supported by NSF Grant No. PHY-8714654. S.D. was supported in part by the U.S. Department of Energy under Grant No. DE-FG02-84ER40158 with Harvard University.
*Present address: The Institute for Advanced Study, Princeton, NJ 08540.

†Present address: NASA/Fermilab Astrophysics Center, Fermilab, Batavia, IL 60510.

[1] C. W. Bernard, Phys. Rev. D 9, 3312 (1974); L. Dolan and R. Jackiw, ibid. 9, 3320 (1974); S. Weinberg, ibid. 9, 3357 (1974). For a clear explanation of the meaning and derivation of the effective potential, see S. Coleman and E. Weinberg, ibid. 7, 1888 (1973); R. Jackiw, ibid. 9, 1686 (1974).

[2] J. I. Kapusta, Phys. Rev. D 24, 426 (1981); H. E. Haber and H. A. Weldon, Phys. Rev. Lett. 46, 1497 (1981); Phys. Rev. D 25, 502 (1982); J. I. Kapusta, Finite Temperature Field Theory (Cambridge University Press, Cambridge, England, 1989).

[3] J. Bernstein and S. Dodelson, Phys. Rev. Lett. 66, 683 (1991).

[4] R. K. Pathria, Statistical Mechanics (Pergamon, Oxford,
1988), Chaps. 7 and 11.

[5] S. Dodelson, B. R. Greene, and D. Spector (unpublished).

[6] See, for example, T. P. Cheng and L. F. Li, Gauge Theory of Elementary Particles (Oxford University Press, New York, 1984); or J. Bernstein, Elementary Particles and Their Currents (Freeman, San Francisco, 1968).

[7] M. A. Beg and R. C. Furlong, Phys. Rev. D 31, 1370 (1985).

[8] R. Jackiw (unpublished).

[9] T. D. Lee and C. N. Yang, Phys. Rev. 105, 1119 (1957).

[10] T. D. Lee, K. Huang, and C. N. Yang, Phys. Rev. 106, 1135 (1957).

[11] T. T. Wu, Phys. Rev. 115, 1390 (1959).

[12] K. Benson and L. Widrow, Nucl. Phys. B353, 187 (1991).

[13] S. Weinberg, Phys. Rev. D 9, 3357 (1974).

[14] P. Fendley, Phys. Lett. B 196, 175 (1987). 